\title{
Tandem Ring Opening/Intramolecular [2 + 2] Cycloaddition Reaction for the Synthesis of Cyclobutane Fused Thiazolino-2- Pyridones
}

\author{
Mohit Tyagi, ${ }^{\S}$ Dan E. Adolfsson, ${ }^{\S}$ Pardeep Singh, ${ }^{\S}$ Jörgen Ådén, Sanduni Wasana Jayaweera, \\ Anna Gharibyan, Jaideep B. Bharate, Anita Kiss, Souvik Sarkar, Anders Olofsson, and Fredrik Almqvist*
}

Cite This: J. Org. Chem. 2021, 86, 16582-16592

Read Online

ACCESS I Lلlll Metrics \& More | 回 Article Recommendations | st Supporting Information

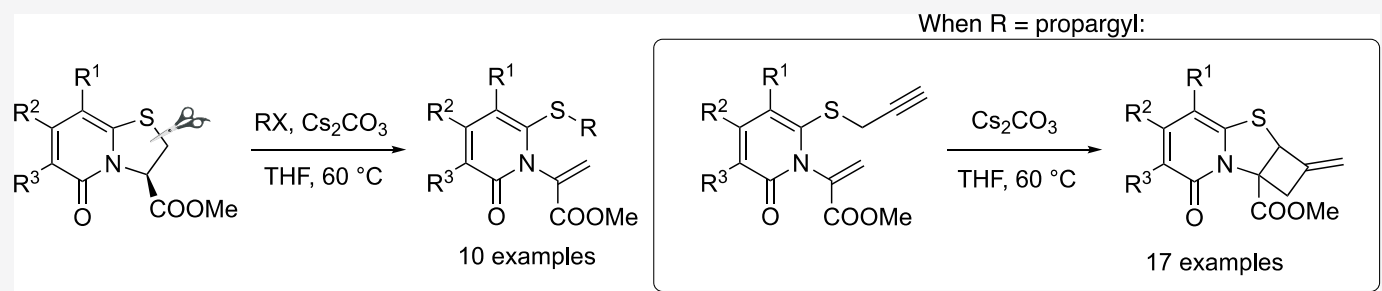

ABSTRACT: Reaction of thiazoline fused 2-pyridones with alkyl halides in the presence of cesium carbonate opens the thiazoline ring via $S$-alkylation and generates $N$-alkenyl functionalized 2-pyridones. In the reaction with propargyl bromide, the thiazoline ring opens and subsequently closes via a $[2+2]$ cycloaddition between an in situ generated allene and the $\alpha, \beta$-unsaturated methyl ester. This method enabled the synthesis of a variety of cyclobutane fused thiazolino-2-pyridones, of which a few analogues inhibit amyloid $\beta_{1-40}$ fibril formation. Furthermore, other analogues were able to bind mature $\alpha$-synuclein and amyloid $\beta_{1-40}$ fibrils. Several thiazoline fused 2-pyridones with biological activity tolerate this transformation, which in addition provides an exocyclic alkene as a potential handle for tuning bioactivity.

\section{INTRODUCTION}

The direct modification of an existing bioactive scaffold rather than the positioning of substituents is an important strategy to develop compounds with diverse shapes and properties. ${ }^{1}$ Cyclobutanes are an important class of rigid motifs present in a variety of natural products and other biologically important molecules. ${ }^{2}$ A plethora of reactions like $[2+2]$ cycloadditions $^{2 \mathrm{c}-\mathrm{e}, 3}$ and rearrangements ${ }^{4}$ have been developed to construct structurally diverse cyclobutane containing scaffolds. Due to their rigid architecture, annulation of a cyclobutane ring with biologically relevant scaffolds like 2-pyridones, ${ }^{5}$ quinolones, ${ }^{6}$ and indoles ${ }^{2 c}$ has recently become popular (Figure 1).

Thiazoline fused 2-pyridones have found various applications in developing biologically active compounds against Escherichia coli, Chlamydia trachomatis, Listeria monocytogenes,
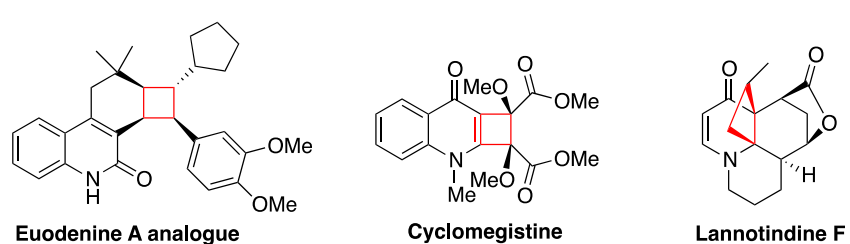

Figure 1. Selected bioactive compounds containing a fused cyclobutane motif. and Mycobacterium tuberculosis infections. ${ }^{7}$ We have also demonstrated that rigidification, either by functionalizing the compounds with sterically demanding aryl groups or annulation with heterocycles, has resulted in ring fused 2pyridones capable of modulating or binding amyloid fibrils. ${ }^{8}$ In a recent report, we demonstrated that the thiazoline ring can be opened by reaction with an aryne to generate $N$-alkenyl-2pyridones (Scheme 1). ${ }^{8 \mathrm{e}}$ Knowing that ring opening results in the formation of a Michael acceptor, we envisaged that reaction of thiazolino-2-pyridones with alkyl halides would generate $N$-alkenyl-S-alkyl-2-pyridones, which could be used as synthons to build structurally diverse scaffolds.

We further envisioned that annulation of the thiazolino-2pyridone scaffold with a cyclobutane ring would help in finetuning biological activity and may result in improved amyloid binding/modulating properties of the resulting compounds. Intramolecular $[2+2]$ cycloadditions of allenes with alkenes constitute a versatile method to synthesize cyclobutane

Received: August 6, 2021

Published: November 12, 2021 
Scheme 1. Electrophilic Thiazoline Ring Opening and Its Application in Synthesizing a Variety of Substituted 2Pyridones $^{a}$

a) Previous work: Electrophilic thiazolino ring opening by thioether attack on aryne. ${ }^{8 \mathrm{e}}$

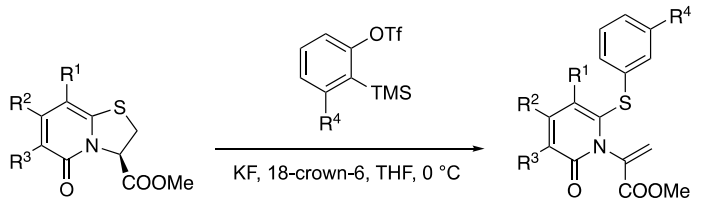

Org. Lett. 2019, 21, 6946-6950

b) This work: Domino ring opening, [2+2] cycloaddition.

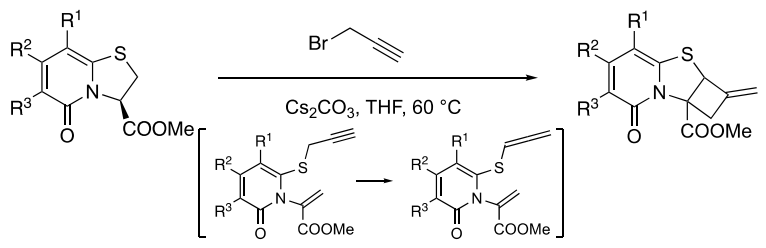

a (a) Previous work. Aryne induced ring opening. (b) This work. Propargyl bromide triggered ring opening followed by thermal $[2+2]$ ring closing cycloaddition.

containing rigid bicyclic frameworks. ${ }^{9}$ Since allenes can be prepared from $S$-propargyls, ${ }^{10}$ we planned to open the thiazoline ring with propargyl halides. The resulting $N$ alkenyl-S-propargyl-2-pyridone could then be used as a building block to construct a cyclobutane fused thiazolino-2pyridone via formation of an allene and a subsequent intramolecular $[2+2]$ cycloaddition.

\section{RESULTS AND DISCUSSION}

To develop the thiazoline ring opening reaction, we commenced our studies by investigating the reaction of 1 a with simple alkyl halides such as methyl iodide. A few bases and solvents were screened to open the ring with methyl iodide (Scheme S1, Supporting Information). Under established conditions, ring opened product $2 \mathrm{a}$ could be obtained in $88 \%$ yield by using $\mathrm{Cs}_{2} \mathrm{CO}_{3}$ in THF at $60{ }^{\circ} \mathrm{C}$ for $24 \mathrm{~h}$ (Scheme $2)$. To further extend the scope, different alkyl halides and substituted 2-pyridones $\mathbf{1 a}-\mathbf{d}$ and $3 \mathbf{3}$ were tested under these standardized conditions to give ring opened 2-pyridones $\mathbf{2 a}-\mathbf{g}$ and 4.

Next, we attempted ring opening of 1a with propargyl bromide (Scheme 3). Pleasingly, cyclobutane fused thiazolino2 -pyridone 5 a was formed in $44 \%$ yield (as a mixture of enantiomers) together with ring opened product $\mathbf{2 h}$ in $20 \%$ yield. To our delight, prolonged heating and use of 3 equiv of $\mathrm{Cs}_{2} \mathrm{CO}_{3}$ gave 5 a exclusively, in $69 \%$ yield (Scheme 4 ). Only starting material was recovered when the reaction was performed in the presence of $\mathrm{Na}_{2} \mathrm{CO}_{3}$ or DIPEA (Scheme S3, Supporting Information). Purified $\mathbf{2 h}$, when treated with $\mathrm{Cs}_{2} \mathrm{CO}_{3}$ in THF, provided 5a, which confirms the intermediacy of $\mathbf{2 h}$.

To evaluate the effect of substituents on the outcome of the reaction, a series of substituted bicyclic thiazolino-2-pyridones was prepared and investigated for their reaction with propargyl bromide (Scheme 4). Compound $\mathbf{5 a - d}$ was provided in moderate to good yield. Substrates equipped with an aryl/ heteroaryl group as $\mathrm{R}^{3}$ substituents reacted smoothly with propargyl bromide to afford 2-pyridones $\mathbf{5 e - h}$ in good yields. In line with our previous study, ${ }^{8}$ low to moderate yields were obtained of $\mathbf{5 i - 1}$, with $\mathrm{CH}_{2}$-naphthyl groups as $\mathrm{R}^{2}$ substituents.
Scheme 2. Ring Opening of Thiazolino-2-pyridones with Alkyl Halides ${ }^{g}$

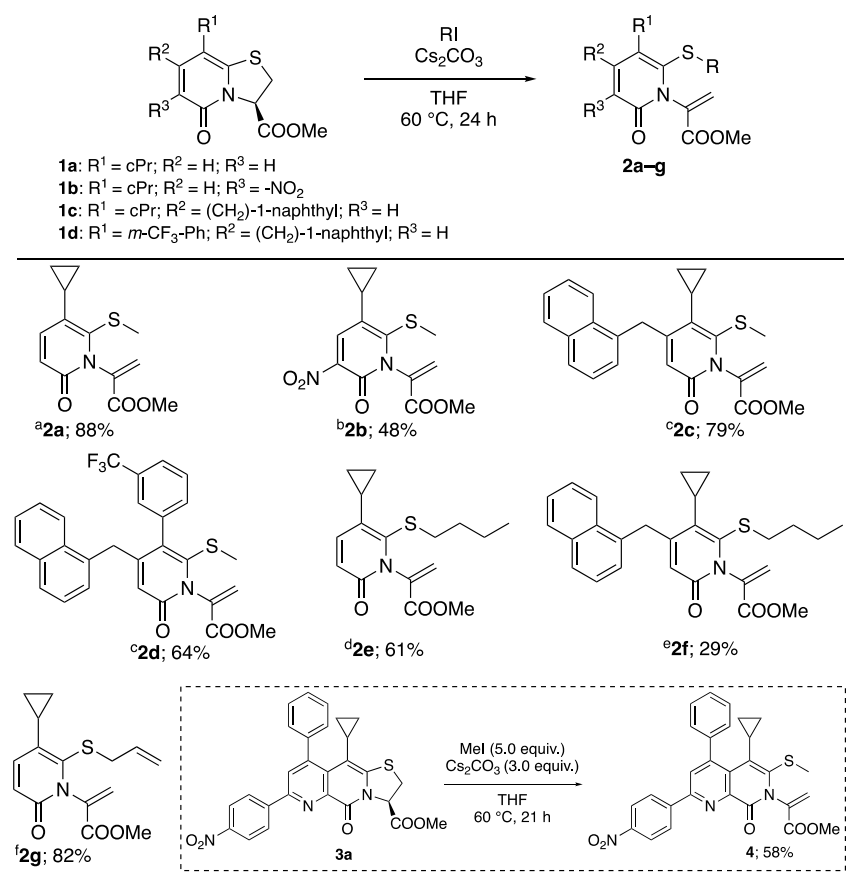

${ }^{a} 3.0$ equiv of methyl iodide. ${ }^{b} 4.2$ equiv of methyl iodide. ${ }^{c} 9.0$ equiv of methyl iodide. ${ }^{d} 47$ h. ${ }^{e} 9.0$ equiv of butyl iodide, 7 days. ${ }^{f} 4.1$ equiv of allyl iodide. ${ }^{g}$ All reactions were performed on a $0.5 \mathrm{mmol}$ scale.

Scheme 3. Ring Opening of 1a with Propargyl Bromide
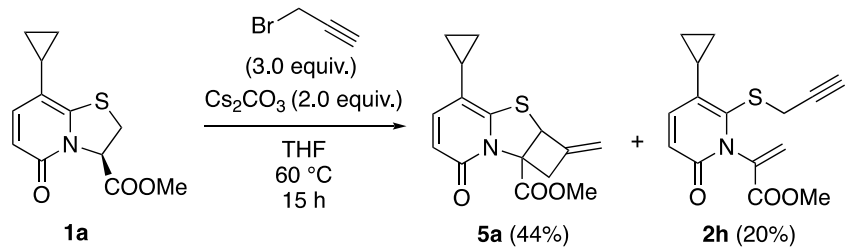

A single crystal X-ray diffraction analysis of analogue 5e verified the structure elucidated by NMR spectroscopy (Scheme 4). When propargyl bromide was replaced with 3bromo-1-butyne or 4-bromo-1-butyne, no ring opening was triggered. With 1-bromo-2-butyne, only the ring opened product $2 \mathbf{i}$ was provided; no further ring closing was observed (Scheme S4, Supporting Information).

The developed intramolecular $[2+2]$ cycloaddition between an in situ generated allene and the $\alpha, \beta$-unsaturated methyl ester gave products as racemic mixtures. To improve diastereoselectivity, sterically demanding chiral esters were prepared using $S$-phenylethanol and menthol. Unfortunately, chiral ester $1 \mathrm{~m}$ derived from $S$-(-)-phenylethanol did not influence the diastereoselectivity and cyclobutane fused thiazoloino-2-pyridone $\mathbf{5 m}$ was isolated as a $1: 1$ diastereomeric mixture (Scheme 5). When L-menthol ester was used as a chiral auxiliary, no ring opening/closing was observed under our standardized conditions (Scheme S5, Supporting Information).

Mechanistically, we propose that nucleophilic attack by the sulfur on propargyl bromide results in the formation of intermediate A (Scheme 6) which upon deprotonation by base gives ring opened product $\mathbf{2 h}$. The intermediate $\mathbf{2 h}$ was isolated and characterized by NMR spectroscopy. Since $S$ - 
Scheme $4^{a}$

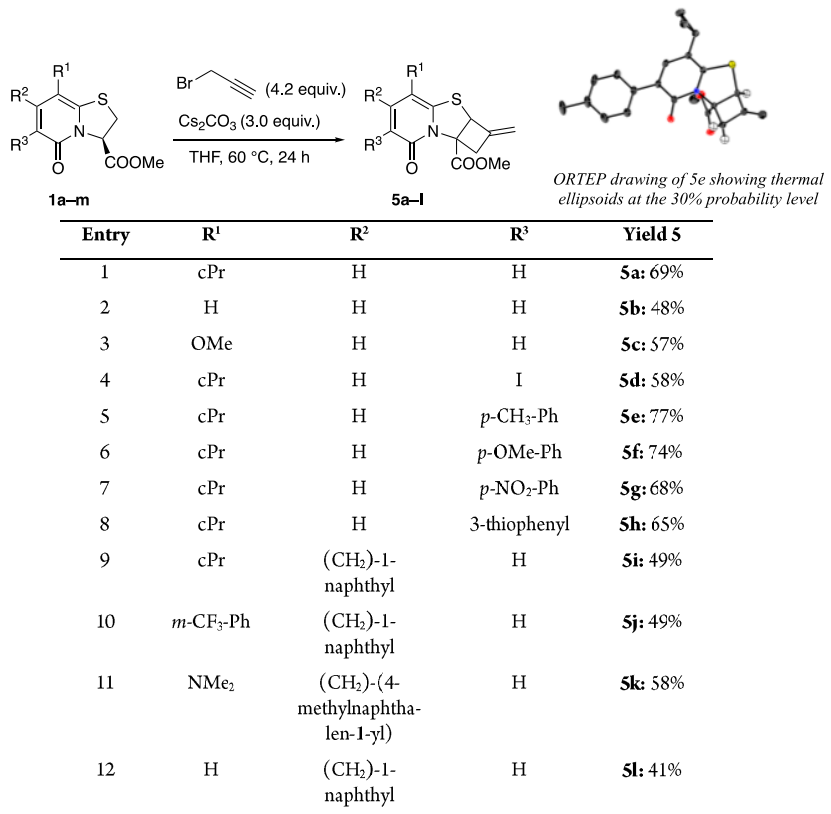

${ }^{a}$ All reactions were performed with $0.5 \mathrm{mmol}$ of 1 at $0.3 \mathrm{M}$ in dry THF. Initially, for $23 \mathrm{~h}, 2.0$ equiv of $\mathrm{Cs}_{2} \mathrm{CO}_{3}$ was added, followed by addition of another 1.0 equiv required for reaction completion. 5e was crystallized from absolute ethanol and obtained as a racemate.

Scheme 5. Tandem Ring Opening/Intramolecular $[2+2]$ Cycloaddition Using Chiral Ester
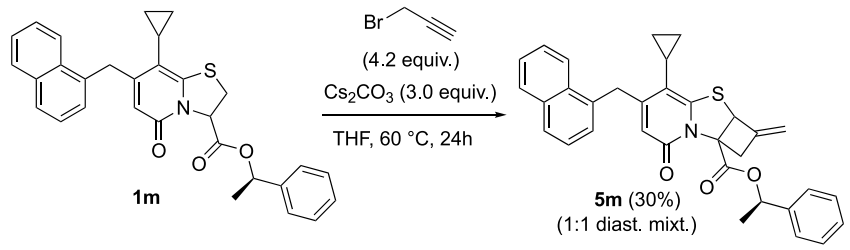

Scheme 6. Tentative Mechanism for the Propargyl Bromide Triggered Ring Opening and Subsequent Intramolecular [2 $+2]$ Cycloaddition

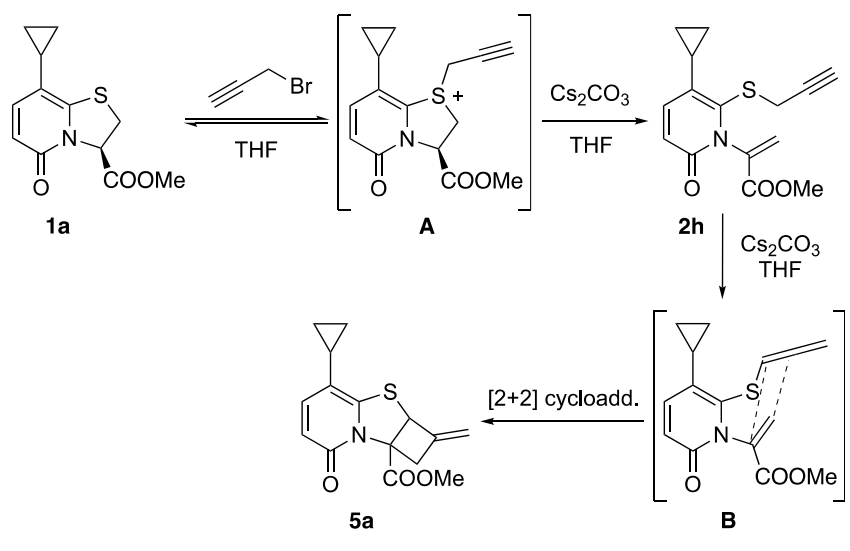

propargyls are known to form allene under basic conditions, ${ }^{10}$ it is likely that base promoted abstraction of methylene protons generates allene $\mathbf{B}$, which undergoes intramolecular $[2+2]$ cycloaddition with the alkene to furnish cyclobutane fused thiazoloino-2-pyridone 5 a.
Knowing that bicyclic and tricyclic thiazolino-2-pyridones have the potential to modulate and bind amyloid fibrils, respectively, cyclobutane fused compounds $5 \mathbf{i}-\mathbf{k}$ and ring opened 2-pyridones $\mathbf{2 c}, \mathbf{2 f}$, and $\mathbf{4}$ were hydrolyzed to their corresponding acids $\mathbf{6 a}-\mathbf{c}, \mathbf{9 a}-\mathbf{b}$, and 7 , respectively (Scheme 7).

\section{Scheme 7. Hydrolysis of Methyl Esters}

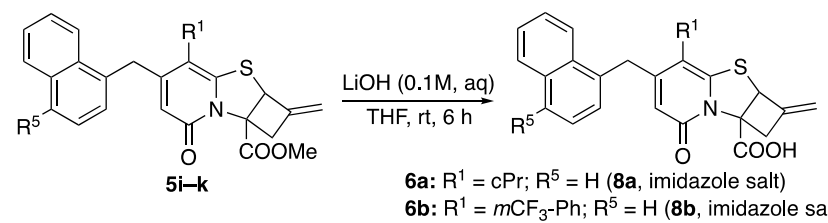

6b: $\mathbf{R}^{1}=m \mathrm{CF}_{3}-\mathrm{Ph} ; \mathrm{R}^{5}=\mathrm{H}(\mathbf{8 b}$, imidazole salt $)$<smiles></smiles>

2c: $R=-M e$

2f: $\mathrm{R}^{1}=n$-butyl

$$
\begin{aligned}
& \text { 9a: } \mathrm{R}=\mathrm{Me} \\
& \text { 9b: } \mathrm{R}=n \text {-butyl }
\end{aligned}
$$
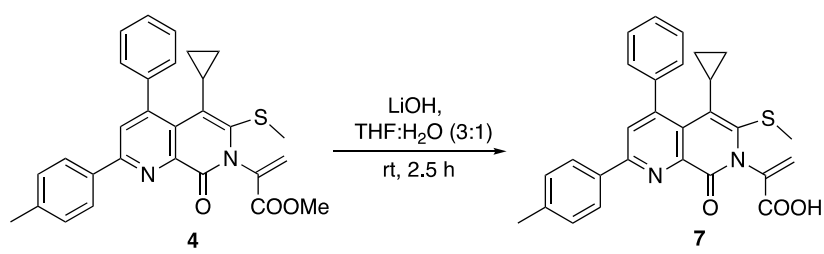

Tricyclic thiazolino-2-pyridones are of therapeutic and diagnostic interest because they have been shown to bind mature $\alpha$-synuclein and $\mathrm{A} \beta$ fibrils. ${ }^{8 \mathrm{~d}}$ Reaction of tricyclic compounds with propargyl bromide (Scheme 8), however,

Scheme 8

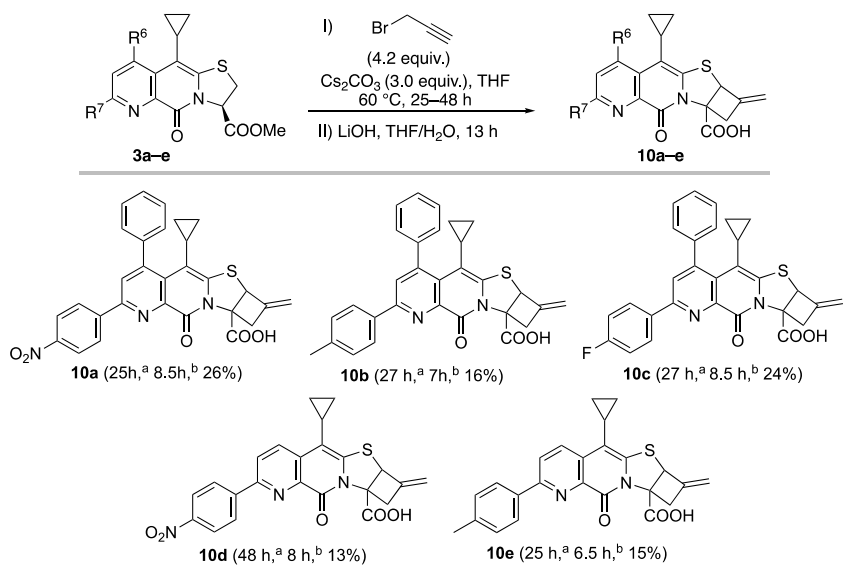

${ }^{a}$ Reaction time for ring opening-closing. ${ }^{b}$ Reaction time for ester hydrolysis. ${ }^{c}$ All reactions were performed on a $0.25 \mathrm{mmol}$ scale at 0.3 $\mathrm{M}$ in dry THF. The mixed esters were, upon purification, directly hydrolyzed to carboxylic acids $10 a-10 e$.

resulted in complex mixtures and the desired cyclobutane fused products were isolated as mixtures of propargyl and methyl esters (perhaps by methyl ester hydrolysis followed by reesterification with propargyl alcohol). Thus, the mixed esters were saponified directly, using lithium hydroxide, to give 10ae, in $13-26 \%$ yield over two steps. 
Compounds $\mathbf{8 a - b}$ (Figure 2), 7, 9a-b (Figures S9-S12, Supporting Information), and $\mathbf{1 0 a}-\mathbf{e}$ (Figure 3) were

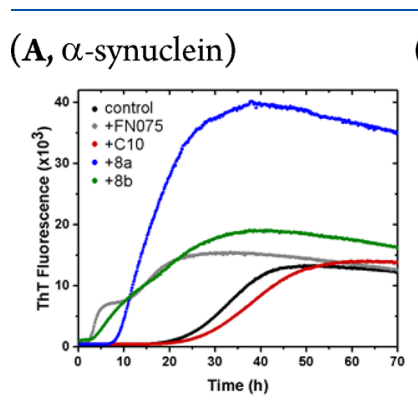

$$
\text { (B, } \left.A \beta_{1-40}\right)
$$
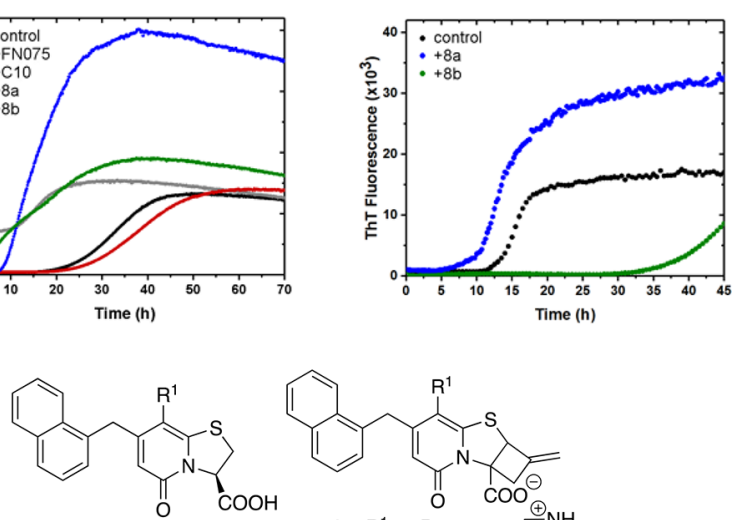

C10: $R^{1}=\mathrm{CPr}$

FNO75: $\mathrm{R}^{1}=m-\mathrm{CF}^{3}-\mathrm{Ph}$

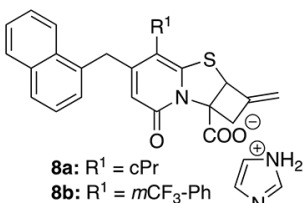

Figure 2. Evaluation of compounds $\mathbf{8 a}$ and $\mathbf{8 b}$ for their effect against (A) $\alpha$-synuclein and (B) amyloid $\beta_{1-40}$ fibril formation in vitro. In the $\alpha$-synuclein assay, compound 8a displays a ThT fluorescence amplitude higher than the control. Both compounds were investigated for whether they modulate the fibers directly, causing the ThT signal to shift. No effect of fiber modulation was found. The higher amplitude seems instead to be a result of altered binding of ThT to the fiber (Figure S16). For control, $\alpha$-synuclein/amyloid $\beta_{1-40}$ was incubated in the absence of 2-pyridone.
(A, $\alpha$-synuclein)

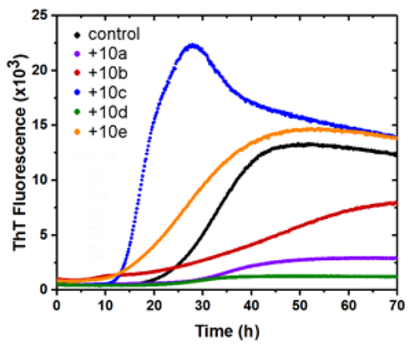

$\left(\right.$ B, $\left.\mathrm{A} \beta_{1-40}\right)$

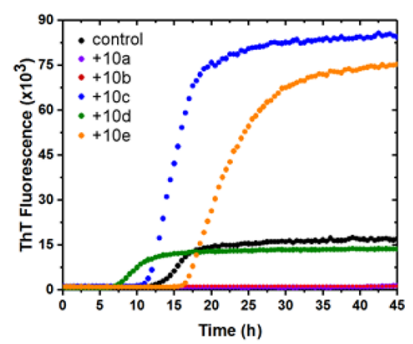

Figure 3. Evaluation of compounds 10a-10e for modulation of (A) $\alpha$-synuclein and (B) amyloid $\beta_{1-40}$ fibril formation in vitro. For control, $\alpha$-synuclein/amyloid $\beta_{1-40}$ was incubated in the absence of 2pyridone.

evaluated for their ability to modulate/bind to $\alpha$-synuclein and amyloid $\beta_{1-40}$ fibrils in vitro. ${ }^{8 \mathrm{~d}}$ In this assay, the effects on fibril formation are observed as changes of the lag phase duration. Further, the ability to bind $\alpha$-Syn fibrils and displace fibril bound ThT is indicated by a reduced ThT fluorescence amplitude in comparison to the control experiments, where no peptidomimetic compound is included. Interestingly, both 8a and $\mathbf{8 b}$ were found to accelerate $\alpha$-synuclein fibril formation, as indicated by reduction of the lag time (Figure 2A). Compound $\mathbf{8 b}$, like its parent compound FN075, showed strong acceleration of $\alpha$-synuclein amyloid formation. The cyclopropyl substituted analogue $\mathbf{8 a}$ displayed a milder accelerating effect, while its parent compound $\mathbf{C 1 0}$ is inactive. ${ }^{8 \mathrm{c}}$ When tested against amyloid $\beta$ fibril formation, $\mathbf{8 b}$ inhibited the formation of fibrils like its parent analogue. ${ }^{8 a}$

Compounds $10 \mathrm{a}-\mathrm{e}$ were tested for their effect against $\alpha$ synuclein and amyloid $\beta_{1-40}$ fibril formation (Figure 3). 4-
Nitrophenyl substituted pyridine fused compounds 10a and 10d, like their parent analogues, ${ }^{8 \mathrm{~d}}$ were found to bind mature $\alpha$-synuclein amyloid fibrils. However, compounds 10b, 10c, and 10e were also found to be very mild accelerators of $\alpha$ synuclein fibril formation (Figure 3A).

Interestingly, when these compounds were tested for their effect against amyloid $\beta_{1-40}$ fibril formation (Figure 3B), compounds $10 \mathbf{a}-\mathbf{b}$ turned out, contrary to their parent compounds which are inactive, to be inhibitors. ${ }^{8 \mathrm{~d}}$

All of the cyclobutane fused thiazolino-2-pyridones were tested as racemates. To investigate the effect of each enantiomer on fibril formation, racemic $\mathbf{6} \mathbf{b}$ was separated to its pure enantiomers using chiral HPLC. When evaluated for their effect on fibril formation in vitro, the pure enantiomers were found to modulate $\alpha$-synuclein and $\mathrm{A} \beta$ fibrils equally, to a similar extent as the racemic mixture (Figures S13 and S14, Supporting Information).

\section{CONCLUSION}

In conclusion, we have prepared $N$-alkenyl 2-pyridones via a thiazoline ring opening reaction with alkyl halides. Reaction of thiazolino-2-pyridones with propargyl bromide gave cyclobutane fused thiazolino-2-pyridones via sequential ring opening, in situ allene formation, and intramolecular $[2+2]$ cycloaddition. The methodology was also successfully applied to functionalize bioactive tricyclic pyridine fused thiazolino-2pyridones. The developed methodology transformed inactive compounds to inhibitors of amyloid $\beta_{1-40}$ fibril formation. Selective modulation of amyloid fibrils by small molecules provides a possible approach in the diagnosis and/or treatment of neurodegenerative diseases, ${ }^{11}$ justifying the importance of such late-stage transformations on thiazolino-2-pyridone peptidomimetic scaffolds for tuning their biological activity. Further advanced structural modifications on these compounds will become a subject for future investigations in order to find new diagnostic/therapeutic agents for neurodegenerative diseases.

\section{EXPERIMENTAL SECTION}

General Information. All reagents were purchased from commercial suppliers and used as received, unless otherwise stated. Molecular sieves were dried at $300{ }^{\circ} \mathrm{C}$ under a high vacuum for $4 \mathrm{~h}$ prior to use. DMF and THF were dried using an SG Water solvent drying tower, according to the manufacturer's instructions, and stored over activated $3 \AA$ (DMF) or $4 \AA$ (THF) molecular sieves $(5 \% \mathrm{w} / \mathrm{v})$ for $48 \mathrm{~h}$ or more before use. $\mathrm{Cs}_{2} \mathrm{CO}_{3}$ was used as purchased from Sigma-Aldrich (i.e., without further drying). Microwave reactions were performed in sealed vessels using a Biotage Initiator microwave synthesizer, temperatures were monitored by an internal IR probe, and stirring was mediated magnetically. TLC was performed on purchased aluminum backed silica gel plates (median pore size $60 \AA$, fluorescent indicator $254 \mathrm{~nm}$ ) and detected with UV light at 254 and $366 \mathrm{~nm}$. Flash column chromatography was performed using silica gel (0.063-0.200 mesh). Automated flash column chromatography was performed using a Biotage Isolera One system and purchased prepacked silica gel cartridges (Biotage SNAP cartridge, KP-Sil or Biotage Sfär Silica D, Duo $60 \mu \mathrm{m}$, cartridge). Preparative HPLC was performed on a Gilson instrument with a Phenomenex column $(250$ $\times 21.2 \mathrm{~mm}^{2}$; Gemini $5 \mu \mathrm{m} \mathrm{NX}-\mathrm{C} 18,110 \AA$ ). $\mathrm{MeCN} /$ water, with $0.1 \%$ $\mathrm{HCOOH}$, was used as mobile phase. A gradient from $30-100 \%$ $\mathrm{MeCN}$ in water was run over $30 \mathrm{~min}$ with a flow rate of $20 \mathrm{~mL} / \mathrm{min}$. The elution was monitored with UV-abs. at $254 \mathrm{~nm}$. Freeze-drying was accomplished by freezing the diluted $\mathrm{MeCN}$ /water solutions in liquid nitrogen and then emloying a Scanvac CoolSafe freeze-dryer connected to an Edwards 28 rotary vane oil pump. IR spectra were 
recorded on a Bruker Alpha-t spectrometer. The samples were prepared as $\mathrm{KBr}$ pellets or between $\mathrm{NaCl}$ plates; absorbances are given in reciprocal $\mathrm{cm} .{ }^{1} \mathrm{H}$ and ${ }^{13} \mathrm{C}$ NMR spectra were recorded on a Bruker Avance III $400 \mathrm{MHz}$ spectrometer with a BBO-F/H Smartprobe or a Bruker Avance III HD $600 \mathrm{MHz}$ spectrometer with a CP BBO-H/F, $5 \mathrm{~mm}$ cryoprobe, at $298 \mathrm{~K}$, unless another temperature is given. All spectrometers were operated by Topspin 3.5.7. Spectra were then processed by MestReNnova v. 10 . Resonances are given in ppm relative to TMS and calibrated to solvent residual signals $\left[\mathrm{CDCl}_{3}: \delta_{\mathrm{H}}=7.26 \mathrm{ppm}, \delta_{\mathrm{C}}=77.16 \mathrm{ppm}\right.$; $\left.\left(\mathrm{CD}_{3}\right)_{2} \mathrm{SO}: \delta_{\mathrm{H}}=2.50 \mathrm{ppm}, \delta_{\mathrm{C}}=39.51 \mathrm{ppm}\right]$. The following abbreviations are used to indicate splitting patterns: $\mathrm{s}=$ singlet; $\mathrm{d}=$ doublet; $\mathrm{dd}=$ double doublet; $\mathrm{t}=$ triplet; $\mathrm{m}=$ multiplet; $\mathrm{bs}=$ broad singlet. LC-MS was conducted on a Micromass ZQ mass spectrometer with $\mathrm{ES}^{+}$and $\mathrm{ES}^{-}$ionization. HRMS was performed on a mass spectrometer with ESI-TOF $\left(\mathrm{ES}^{+} / \mathrm{ES}^{-}\right)$. Bicyclic 2pyridones $1 \mathbf{a}-\mathbf{d}$ and tricyclic 2-pyridones $3 \mathbf{a}-\mathbf{e}$ were prepared according to the reported procedures. ${ }^{7,8 d, e}$ An Oxford Diffraction Excalibur 3 system was used for X-ray data collection and Crysalis RED data extraction. Crystal Maker 9.2 was used for molecular graphics.

General Procedure for Synthesis of $\mathbf{2 a - g}$ and 4. Thiazolino fused 2-pyridone 1 ( $0.5 \mathrm{mmol}, 1.0$ equiv) and cesium carbonate $(326 \mathrm{mg}$, $1.0 \mathrm{mmol}, 2.0$ equiv) were weighed in an oven-dried Biotage Initiator microwave reaction tube $(2-5 \mathrm{~mL})$ equipped with a magnetic follower. The tube was sealed with a septum and put under a high vacuum for $30 \mathrm{~min}$ at room temperature and then backfilled with nitrogen. Dried THF $(1.5 \mathrm{~mL})$ was added with a syringe. The septum was removed briefly to add alkyl halide (3.0-9.0 equiv) with an automatic pipet, and the tube was quickly sealed with a crimp cap. The resulting suspension was stirred in an oil bath at $60{ }^{\circ} \mathrm{C}$ until reaction completion was indicated. The reactions were monitored with TLC on samples extracted with syringes. Upon complete consumption of starting material $\mathbf{1}$, the reaction mixture was transferred to a separation funnel and partitioned between brine $(25 \mathrm{~mL})$ and EtOAc $(2 \times 25 \mathrm{~mL})$. The organic phases were combined, dried over anhydrous sodium sulfate, filtered, and evaporated. The residue was redissolved in a small amount of DCM and purified with automated flash column chromatography.

(R)-1-Phenylethyl (R)-8-Cyclopropyl-7-(naphthalen-1-ylmethyl)5-oxo-2,3-dihydro-5H-thiazolo[3,2-a]pyridine-3-carboxylate (1 m). C-10 (200 mg, $0.529 \mathrm{mmol})$, DMAP $(6.47 \mathrm{mg}, 0.053 \mathrm{mmol})$, and DCC (163 mg, $0.794 \mathrm{mmol})$ were dissolved in DCM $(5 \mathrm{~mL})$ at 25 ${ }^{\circ} \mathrm{C}$, and $(S)-(-)-1$-phenylethanol $(100 \mu \mathrm{L}, 0.834 \mathrm{mmol})$ was added dropwise to the mixture. The reaction mixture was then left stirring at $40{ }^{\circ} \mathrm{C}$ overnight. After $24 \mathrm{~h}$, the reaction mixture was diluted with DCM $(100 \mathrm{~mL})$, washed with aqueous $\mathrm{NH}_{4} \mathrm{Cl}$ (saturated) followed by washing with brine $(150 \mathrm{~mL})$, and dried over anhydrous $\mathrm{Na}_{2} \mathrm{SO}_{4}$, filtered, and concentrated. The crude product was purified by automated flash column chromatography (25 g SNAP cartridge) eluting with $0-40 \%$ ethyl acetate in heptane, to provide $170 \mathrm{mg}$ $(67 \%)$ of $1 \mathrm{~m}$ as a white powder. IR (KBr): $\nu 3450,1740,1654,1579$, $1487,1424,1285,1209,1160,1090,1061,1028,993,780,760 \mathrm{~cm}^{-1}$. ${ }^{1} \mathrm{H}$ NMR $\left(400 \mathrm{MHz}, \mathrm{CDCl}_{3}\right) \delta 7.79(\mathrm{dd}, J=7.7,1.7 \mathrm{~Hz}, 1 \mathrm{H}), 7.76-$ $7.62(\mathrm{~m}, 2 \mathrm{H}), 7.44-7.29(\mathrm{~m}, 3 \mathrm{H}), 7.24-7.16(\mathrm{~m}, 5 \mathrm{H}), 5.85(\mathrm{~d}, J=$ $6.6 \mathrm{~Hz}, 1 \mathrm{H}), 5.69(\mathrm{~d}, J=7.8 \mathrm{~Hz}, 1 \mathrm{H}), 5.50(\mathrm{dd}, J=8.6,2.3 \mathrm{~Hz}, 1 \mathrm{H})$, 4.34 (dd, $J=40.0,17.2 \mathrm{~Hz}, 2 \mathrm{H}), 3.55(\mathrm{dd}, J=11.7,8.6 \mathrm{~Hz}, 1 \mathrm{H}), 3.33$ (dd, $J=11.7,2.3 \mathrm{~Hz}, 1 \mathrm{H}), 1.81$ (dd, $J=12.6,3.4 \mathrm{~Hz}, 1 \mathrm{H}), 1.63-1.52$ $(\mathrm{m}, 2 \mathrm{H}), 1.50(\mathrm{t}, J=5.9 \mathrm{~Hz}, 3 \mathrm{H}), 1.43(\mathrm{~d}, J=6.6 \mathrm{~Hz}, 1 \mathrm{H}), 1.26-1.20$ $(\mathrm{m}, 1 \mathrm{H}), 1.06-1.00(\mathrm{~m}, 1 \mathrm{H}), 0.88-0.75(\mathrm{~m}, 2 \mathrm{H}), 0.63(\mathrm{td}, J=6.0$, $3.0 \mathrm{~Hz}, 2 \mathrm{H}) ;{ }^{13} \mathrm{C}\left\{{ }^{1} \mathrm{H}\right\}$ NMR $\left[100 \mathrm{MHz}, \mathrm{CDCl}_{3}\right] \delta 167.6,161.5$, $156.9,147.2$, 140.9, 134.1, 132.1, 129.0, 128.7, 128.6, 128.2, 127.8, 127.6, 126.4, 126.1, 125.8, 125.7, 123.9, 115.5, 113.6, 74.8, 63.1, 36.4, 32.1, 25.7, 22.2, 11.4, 8.0; HRMS (ESI-TOF) $\mathrm{m} / z[\mathrm{M}+\mathrm{H}]^{+}$calcd for $\mathrm{C}_{30} \mathrm{H}_{28} \mathrm{NO}_{3} \mathrm{~S}^{+}$482.1790; found 482.1795 .

Methyl 2-(5-Cyclopropyl-6-(methylthio)-2-oxopyridin-1(2H)-yl)acrylate (2a). The compound was prepared from 1 a $(118 \mathrm{mg}, 0.47$ $\mathrm{mmol}$ ) following the general procedure, using 3.0 equiv of methyl iodide $(88 \mu \mathrm{L}, 1.4 \mathrm{mmol})$. The reaction was complete to TLC analysis after $23 \mathrm{~h}$. The crude product was purified with automated flash column chromatography (10 g Sfär cartridge, 20-80\% EtOAc in heptane) to give pure $2 \mathrm{a}$ as an orange solid (109 $\mathrm{mg}, 0.412 \mathrm{mmol}$, $88 \%)$. IR (KBr): $\nu$ 3083, 3001, 2952, 2924, 1734, 1669, 1591, 1497, $1437,1363,1328,1302,1250,1199,1170,1087,1038 \mathrm{~cm}^{-1} .{ }^{1} \mathrm{H}$ NMR $\left(400 \mathrm{MHz}, \mathrm{CDCl}_{3}\right) \delta 6.89(\mathrm{~d}, J=9.6 \mathrm{~Hz}, 1 \mathrm{H}), 6.73(\mathrm{~s}, 1 \mathrm{H})$, $6.62(\mathrm{~d}, J=9.6 \mathrm{~Hz}, 1 \mathrm{H}), 5.83(\mathrm{~s}, 1 \mathrm{H}), 3.82(\mathrm{~s}, 3 \mathrm{H}), 2.37-2.30(\mathrm{~m}$, $1 \mathrm{H}), 2.29(\mathrm{~s}, 3 \mathrm{H}), 1.04-0.93(\mathrm{~m}, 2 \mathrm{H}), 0.69-0.60(\mathrm{~m}, 2 \mathrm{H}) .{ }^{13} \mathrm{C}\left\{{ }^{1} \mathrm{H}\right\}$ NMR $\left(100 \mathrm{MHz}, \mathrm{CDCl}_{3}\right) \delta 163.3,162.1,140.9,137.3,136.1,126.9$, $122.8,122.0,52.8,19.6,12.4,7.6,7.4$. HRMS (ESI-TOF) $\mathrm{m} / \mathrm{z}:[\mathrm{M}+$ $\mathrm{H}]^{+}$calcd for $\mathrm{C}_{13} \mathrm{H}_{16} \mathrm{NO}_{3} \mathrm{~S}^{+} 266.0845$; observed 266.0846 .

Methyl 2-(5-Cyclopropyl-6-(methylthio)-3-nitro-2-oxopyridin$1(2 \mathrm{H})$-yl)acrylate (2b). The compound was prepared from $\mathbf{1 b}$ (148 $\mathrm{mg}, 0.5 \mathrm{mmol}$ ) following the general procedure, using 4.2 equiv of methyl iodide (131 $\mu \mathrm{L}, 2.1 \mathrm{mmol})$. The reaction was complete to TLC analysis after $21 \mathrm{~h}$. The crude product was purified with automated flash column chromatography (10 g Sfär cartridge, 10$50 \%$ EtOAc in heptane) to give pure $\mathbf{2 b}$ as a bright yellow solid (75 $\mathrm{mg}, 0.240 \mathrm{mmol}, 48 \%)$. IR (KBr): $\nu$ 3010, 2955, 1733, 1690, 1639, $1516,1483,1438,1392,1308,1235,1201,1170,1093 \mathrm{~cm}^{-1} .{ }^{1} \mathrm{H}$ $\operatorname{NMR}\left(400 \mathrm{MHz}, \mathrm{CDCl}_{3}\right) \delta 7.97(\mathrm{~s}, 1 \mathrm{H}), 6.79(\mathrm{~d}, J=1.2 \mathrm{~Hz}, 1 \mathrm{H})$, $5.89(\mathrm{~d}, J=1.2 \mathrm{~Hz}, 1 \mathrm{H}), 3.84(\mathrm{~s}, 3 \mathrm{H}), 2.44(\mathrm{~s}, 3 \mathrm{H}), 2.30-2.20(\mathrm{~m}$, $1 \mathrm{H}), 1.17-1.05(\mathrm{~m}, 2 \mathrm{H}), 0.78-0.70(\mathrm{~m}, 2 \mathrm{H}) .{ }^{13} \mathrm{C}\left\{{ }^{1} \mathrm{H}\right\}$ NMR $(100$ $\left.\mathrm{MHz}, \mathrm{CDCl}_{3}\right) \delta 162.5,154.0,152.4,138.3,136.9,135.9,128.3,124.0$, 53.3, 19.8, 13.0, 8.8, 8.1. HRMS (ESI-TOF) $\mathrm{m} / z$ : $[\mathrm{M}+\mathrm{H}]^{+}$calcd for $\mathrm{C}_{13} \mathrm{H}_{15} \mathrm{~N}_{2} \mathrm{O}_{5} \mathrm{~S}^{+}$311.0696; observed 311.0691 .

Methyl 2-(5-Cyclopropyl-6-(methylthio)-4-(naphthalen-1-ylmethyl)-2-oxopyridin-1(2H)-yl)acrylate (2c). The compound was prepared from 1c (176 mg, $0.45 \mathrm{mmol}$ ) following the general procedure, using 9.0 equiv of methyl iodide $(252 \mu \mathrm{L}, 4.05 \mathrm{mmol})$. The reaction was complete to TLC analysis after $21 \mathrm{~h}$. The crude product was purified with automated flash column chromatography ( $10 \mathrm{~g}$ Sfär cartridge, $10-60 \%$ EtOAc in heptane) to give pure $2 \mathrm{c}$ as an off white powder $(144 \mathrm{mg}, 0.355 \mathrm{mmol}, 79 \%)$. IR (KBr): $\nu$ 3044, 2999, 2951, 1734, 1663, 1583, 1510,1482, 1437, 1404, 1358, 1331, 1281, 1204, 1178, $1134 \mathrm{~cm}^{-1} .{ }^{1} \mathrm{H}$ NMR $\left[400 \mathrm{MHz},\left(\mathrm{CD}_{3}\right)_{2} \mathrm{SO}\right] \delta$ $8.04-7.93(\mathrm{~m}, 1 \mathrm{H}), 7.89(\mathrm{dd}, J=9.6,6.9 \mathrm{~Hz}, 2 \mathrm{H}), 7.59-7.46(\mathrm{~m}$, $3 \mathrm{H}), 7.42(\mathrm{~d}, J=6.9 \mathrm{~Hz}, 1 \mathrm{H}), 6.55(\mathrm{~s}, 1 \mathrm{H}), 5.92(\mathrm{~s}, 1 \mathrm{H}), 5.49(\mathrm{~s}$, $1 \mathrm{H}), 4.54(\mathrm{~d}, J=8.3 \mathrm{~Hz}, 2 \mathrm{H}), 3.71(\mathrm{~s}, 3 \mathrm{H}), 2.40(\mathrm{~s}, 3 \mathrm{H}), 1.88-1.77$ $(\mathrm{m}, 1 \mathrm{H}), 1.23-0.91(\mathrm{~m}, 3 \mathrm{H}), 0.80-0.70(\mathrm{~m}, 1 \mathrm{H}) .{ }^{13} \mathrm{C}\left\{{ }^{1} \mathrm{H}\right\} \mathrm{NMR}$ $\left[100 \mathrm{MHz},\left(\mathrm{CD}_{3}\right)_{2} \mathrm{SO}\right] \delta 162.9,160.7,156.4,144.6,135.6,134.2$, $133.5,131.6,128.7,128.0,127.8,127.5,126.5,125.9,125.8,124.0$, 123.5, 118.1, 79.2, 52.6, 35.6, 19.8, 11.3, 10.8, 8.9. HRMS (ESI-TOF) $m / z:[\mathrm{M}+\mathrm{H}]^{+}$calcd for $\mathrm{C}_{24} \mathrm{H}_{24} \mathrm{NO}_{3} \mathrm{~S}^{+} 406.1471$; observed 406.1486 .

Methyl 2-(6-(Methylthio)-4-(naphthalen-1-ylmethyl)-2-oxo-5-(3(trifluoromethyl)phenyl)pyridin-1 $(2 \mathrm{H})$-yl)acrylate (2d). The compound was prepared from $1 \mathrm{~d}(248 \mathrm{mg}, 0.5 \mathrm{mmol})$ following the general procedure, using 9.0 equiv of methyl iodide $(280 \mu \mathrm{L}, 4.5$ $\mathrm{mmol}$ ). The reaction was complete to TLC analysis after $21 \mathrm{~h}$. The crude product was purified with automated flash column chromatography (10 g Sfär cartridge, $10-50 \%$ EtOAc in heptane) to give pure 2d as an off white solid (162 mg, $0.317 \mathrm{mmol}, 64 \%)$. IR (KBr): $\nu$ 3046, 3002, 2953, 1734, 1669, 1587, 1500, 1482, 1438, 1401, 1329, $1288,1203,1166,1126,1094,1075,1059 \mathrm{~cm}^{-1}$. ${ }^{1} \mathrm{H}$ NMR $[400 \mathrm{MHz}$, $\left.\left(\mathrm{CD}_{3}\right)_{2} \mathrm{SO}, 343 \mathrm{~K}\right] \delta 7.93-7.86(\mathrm{~m}, 1 \mathrm{H}), 7.81(\mathrm{~d}, J=8.2 \mathrm{~Hz}, 1 \mathrm{H})$, 7.73-7.54 (m, 5H), 7.52-7.40 (m, 3H), $7.24(\mathrm{dd}, J=7.1,1.2 \mathrm{~Hz}$, $1 \mathrm{H}), 6.65(\mathrm{~d}, J=1.0 \mathrm{~Hz}, 1 \mathrm{H}), 6.10(\mathrm{~d}, J=1.0 \mathrm{~Hz}, 1 \mathrm{H}), 5.99(\mathrm{~s}, 1 \mathrm{H})$, 4.00-3.88 (m, 2H) $3.76(\mathrm{~s}, 3 \mathrm{H}), 2.02(\mathrm{~s}, 3 \mathrm{H}) .{ }^{13} \mathrm{C}\left\{{ }^{1} \mathrm{H}\right\}$ NMR $[100$ $\left.\mathrm{MHz},\left(\mathrm{CD}_{3}\right)_{2} \mathrm{SO}, 343 \mathrm{~K}\right] \delta 162.7,160.7,152.6,141.8,137.2,135.0$, $133.1,133.1,130.9,128.9,128.6,128.2,127.5,127.2,127.1,126.3$, 125.9, 125.4, 125.1, 124.0, 123.9, 123.1, 119.8, 78.8, 52.3, 36.2, 19.3. ${ }^{19} \mathrm{~F}$ NMR $\left[376 \mathrm{MHz},\left(\mathrm{CD}_{3}\right)_{2} \mathrm{SO}, 343 \mathrm{~K}\right] \delta-61.2$. HRMS (ESI-TOF) $m / z:[\mathrm{M}+\mathrm{H}]^{+}$calcd for $\mathrm{C}_{28} \mathrm{H}_{23} \mathrm{~F}_{3} \mathrm{NO}_{3} \mathrm{~S}^{+}$510.1345; observed 510.1359 .

Methyl 2-(6-(Butylthio)-5-cyclopropyl-2-oxopyridin-1(2H)-yl)acrylate (2e). The compound was prepared from la (126 mg, 0.5 $\mathrm{mmol}$ ) following the general procedure, using 3.0 equiv of butyl iodide $(171 \mu \mathrm{L}, 1.5 \mathrm{mmol})$. The reaction was complete to TLC analysis after $47 \mathrm{~h}$. The crude product was purified with automated flash column chromatography (10 g Sfär cartridge, 10-70\% EtOAc in heptane) to give pure $2 \mathrm{e}$ as a yellow solid (93 $\mathrm{mg}, 0.303 \mathrm{mmol} 61 \%$ ). 
IR (KBr): $\nu$ 2957, 1736, 1671, 1593, 1498, 1438, 1363, 1327, 1303, 1249, 1200, 1171, $1088 \mathrm{~cm}^{-1}$. ${ }^{1} \mathrm{H}$ NMR $\left(600 \mathrm{MHz}, \mathrm{CDCl}_{3}\right) \delta 6.86$ $(\mathrm{d}, J=9.6 \mathrm{~Hz}, 1 \mathrm{H}), 6.73(\mathrm{~s}, 1 \mathrm{H}), 6.62(\mathrm{~d}, J=9.6 \mathrm{~Hz}, 1 \mathrm{H}), 5.82(\mathrm{~d}, J=$ $0.7 \mathrm{~Hz}, 1 \mathrm{H}), 3.80(\mathrm{~s}, 3 \mathrm{H}), 2.71(\mathrm{q}, J=7.3 \mathrm{~Hz}, 2 \mathrm{H}), 2.35$ (tt, $J=8.5$, $5.2 \mathrm{~Hz}, 1 \mathrm{H}), 1.58-1.48(\mathrm{~m}, 2 \mathrm{H}), 1.42-1.34(\mathrm{~m}, 2 \mathrm{H}), 1.01-0.94(\mathrm{~m}$, $2 \mathrm{H}), 0.89(\mathrm{t}, J=7.3 \mathrm{~Hz}, 3 \mathrm{H}), 0.68-0.61(\mathrm{~m}, 2 \mathrm{H}) .{ }^{13} \mathrm{C}\left\{{ }^{1} \mathrm{H}\right\}$ NMR $\left(151 \mathrm{MHz}, \mathrm{CDCl}_{3}\right) \delta 163.4,162.4,140.0,137.1,136.3,127.6,127.2$, 122.0, 52.9, 36.9, 31.3, 22.1, 13.7, 12.8, 7.9, 7.8. HRMS (ESI-TOF) $m / z:[\mathrm{M}+\mathrm{H}]^{+}$calcd for $\mathrm{C}_{16} \mathrm{H}_{21} \mathrm{NO}_{3} \mathrm{~S}$ 308.1315; observed 308.1328.

Methyl 2-(6-(Butylthio)-5-cyclopropyl-4-(naphthalen-1-ylmethyl)-2-oxopyridin-1(2H)-yl)acrylate (2f). The compound was prepared from 1c (196 mg, $0.5 \mathrm{mmol})$ following the general procedure, using 9.0 equiv of butyl iodide $(280 \mu \mathrm{L}, 4.5 \mathrm{mmol})$. The reaction was complete to TLC analysis after $7 \mathrm{~d}$. The crude product was purified with automated flash column chromatography (25 g Sfär cartridge, $10-40 \%$ EtOAc in heptane) to give pure $2 \mathrm{f}$ as a light yellow solid (66 $\mathrm{mg}, 0.147 \mathrm{mmol}, 29 \%)$. IR (KBr): $\nu$ 3001, 2955, 2931, 2870, 1736, $1665,1584,1481,1435,1403,1358,1331,1280,1203,1178,1134$, 792, $781 \mathrm{~cm}^{-1} .{ }^{1} \mathrm{H}$ NMR $\left(400 \mathrm{MHz}, \mathrm{CDCl}_{3}\right) \delta 7.90-7.84(\mathrm{~m}, 1 \mathrm{H})$, 7.84-7.75 (m, 2H), 7.51-7.44 (m, 2H), 7.44-7.38 (m, 1H), $7.26(\mathrm{~d}$, $J=9.0 \mathrm{~Hz}, 1 \mathrm{H}), 6.67(\mathrm{~s}, 1 \mathrm{H}), 6.03(\mathrm{~s}, 1 \mathrm{H}), 5.76(\mathrm{~s}, 1 \mathrm{H}), 4.58-4.40$ $(\mathrm{m}, 2 \mathrm{H}), 3.79(\mathrm{~s}, 3 \mathrm{H}), 2.83(\mathrm{q}, J=7.5 \mathrm{~Hz}, 2 \mathrm{H}), 1.62-1.46(\mathrm{~m}, 3 \mathrm{H})$, $1.44-1.30(\mathrm{~m}, 2 \mathrm{H}), 1.22-1.12(\mathrm{~m}, 1 \mathrm{H}), 1.11-0.95(\mathrm{~m}, 2 \mathrm{H}), 0.91(\mathrm{t}$, $J=7.3 \mathrm{~Hz}, 3 \mathrm{H}), 0.85-0.74(\mathrm{~m}, 1 \mathrm{H}) .{ }^{13} \mathrm{C}\left\{{ }^{1} \mathrm{H}\right\}$ NMR $(100 \mathrm{MHz}$, $\left.\mathrm{CDCl}_{3}\right) \delta 163.3,162.2,155.9,143.6,136.3,134.1,134.1,132.1,129.0$, $127.9,127.6,127.4,126.4,125.9,125.7,124.6,123.8,120.2,52.8$, 36.9, 36.6, 31.3, 22.0, 13.8, 11.9, 11.5, 9.6. HRMS (ESI-TOF) $\mathrm{m} / z$ : $[\mathrm{M}+\mathrm{H}]^{+}$calcd for $\mathrm{C}_{27} \mathrm{H}_{30} \mathrm{NO}_{3} \mathrm{~S}^{+} 448.1941$; observed 448.1954 .

Methyl 2-(6-(Allylthio)-5-cyclopropyl-2-oxopyridin-1(2H)-yl)acrylate (2g). The compound was prepared from $1 \mathrm{a}(126 \mathrm{mg}, 0.5$ $\mathrm{mmol})$ following the general procedure using allyl iodide $(192 \mu \mathrm{L}, 2.1$ mmol). TLC showed completion of reaction in $12 \mathrm{~h}$. The crude product was purified with automated flash column chromatography (50 g Sfär cartridge, $10-80 \%$ EtOAc in heptane) to give pure $2 \mathrm{~g}$ as a light brown solid (120 mg, $0.4 \mathrm{mmol}, 82 \%)$. IR (KBr): $\nu$ 3435, 3081, 3003, 2953, 1734, 1669, 1592, 1498, 1437, 1326, 1303, 1250, 1200, $1171 \mathrm{~cm}^{-1}$. ${ }^{1} \mathrm{H}$ NMR $\left(400 \mathrm{MHz}, \mathrm{CDCl}_{3}\right) \delta 6.85(\mathrm{~d}, J=9.6 \mathrm{~Hz}, 1 \mathrm{H})$, $6.75(\mathrm{~d}, J=0.7 \mathrm{~Hz}, 1 \mathrm{H}), 6.59(\mathrm{dd}, J=9.6,0.6 \mathrm{~Hz}, 1 \mathrm{H}), 5.87-5.70$ $(\mathrm{m}, 2 \mathrm{H}), 5.11-4.99(\mathrm{~m}, 2 \mathrm{H}), 3.81(\mathrm{~s}, 3 \mathrm{H}), 3.40(\mathrm{dd}, J=12.7,7.1 \mathrm{~Hz}$, $1 \mathrm{H}), 3.29$ (dd, $J=12.7,7.6 \mathrm{~Hz}, 1 \mathrm{H}), 2.40-2.30(\mathrm{~m}, 1 \mathrm{H}), 1.04-0.90$ (m, 2H), 0.72-0.52 (m, 2H). ${ }^{13} \mathrm{C}\left\{{ }^{1} \mathrm{H}\right\}$ NMR (100 MHz, $\left.\mathrm{CDCl}_{3}\right) \delta$ 163.4, 162.1, 138.3, 136.6, 136.1, 132.2, 127.8, 127.3, 122.4, 118.8, 77.3, 77.0, 76.7, 52.8, 39.8, 12.7, 7.8, 7.8. HRMS (ESI-TOF) $\mathrm{m} / z$ : [M $+\mathrm{H}]^{+}$calcd for $\mathrm{C}_{15} \mathrm{H}_{18} \mathrm{NO}_{3} \mathrm{~S}^{+}$292.1002; observed 292.1011.

Methyl 2-(5-Cyclopropyl-6-(methylthio)-2-(4-nitrophenyl)-8-oxo4-phenyl-1,7-naphthyridin-7(8H)-yl)acrylate (4). The compound was prepared from $3 \mathrm{a}(125 \mathrm{mg}, 0.25 \mathrm{mmol})$ following the general procedure but at $0.25 \mathrm{mmol}$ scale, using 5.0 equiv of methyl iodide (78 $\mu \mathrm{L}, 1.25 \mathrm{mmol})$ and 3.0 equiv of cesium carbonate $(244 \mathrm{mg}, 0.75$ $\mathrm{mmol}$ ). The reaction was complete to TLC analysis after $21 \mathrm{~h}$. The crude product was purified with automated flash column chromatography (10 g Sfär cartridge, 10-50\% EtOAc in heptane) to give pure 4 as a bright yellow powder (74 $\mathrm{mg}, 0.144 \mathrm{mmol}, 58 \%)$. IR (KBr): $\nu$ 3080, 3003, 2951, 2926, 1734, 1677, 1585, 1520, 1453, 1438, 1410, $1344,1256,1210,1155,1141,1108,911,853,735 \mathrm{~cm}^{-1} .{ }^{1} \mathrm{H}$ NMR $\left(400 \mathrm{MHz}, \mathrm{CDCl}_{3}\right) \delta 8.42(\mathrm{~d}, J=9.0 \mathrm{~Hz}, 2 \mathrm{H}), 8.33(\mathrm{~d}, J=9.0 \mathrm{~Hz}$, $2 \mathrm{H}), 8.00(\mathrm{~s}, 1 \mathrm{H}), 7.65-7.36(\mathrm{~m}, 5 \mathrm{H}), 6.82(\mathrm{~d}, J=0.7 \mathrm{~Hz}, 1 \mathrm{H}), 6.00$ (d, $J=0.8 \mathrm{~Hz}, 1 \mathrm{H}), 3.84(\mathrm{~s}, 3 \mathrm{H}), 2.42(\mathrm{~s}, 3 \mathrm{H}), 1.27-1.12(\mathrm{~m}, 1 \mathrm{H})$, $0.68-0.13(\mathrm{~m}, 4 \mathrm{H}) .{ }^{13} \mathrm{C}\left\{{ }^{1} \mathrm{H}\right\}$ NMR $\left(100 \mathrm{MHz}, \mathrm{CDCl}_{3}\right) \delta 163.6$, 160.5 , 153.1, 149.4, 148.6, 143.9, 143.8, 143.3, 141.3, 135.7, 133.6, $128.7,128.3,128.3,127.8,126.6,124.1,120.5,53.0,20.2,16.7$. HRMS (ESI-TOF) $m / z:[\mathrm{M}+\mathrm{H}]^{+}$calcd for $\mathrm{C}_{28} \mathrm{H}_{24} \mathrm{~N}_{3} \mathrm{O}_{5} \mathrm{~S}^{+}$ 514.1431; observed 514.1444.

General Procedure for Synthesis of $\mathbf{2 h}$ and $\mathbf{5 a - l}$. Thiazolino fused 2-pyridone 1 ( $0.5 \mathrm{mmol}, 1.0$ equiv) and cesium carbonate (326 $\mathrm{mg}, 1.0 \mathrm{mmol}, 2.0$ equiv) were weighed in an oven-dried $2-5 \mathrm{~mL}$ microwave vial and flushed with nitrogen. Dried THF $(1.5 \mathrm{~mL})$ and propargyl bromide ( $225 \mu \mathrm{L}, 2.10 \mathrm{mmol}, 4.2$ equiv) were added to it, and the resulting mixture was stirred at $60^{\circ} \mathrm{C}$. After $23 \mathrm{~h}$, additional cesium carbonate ( $163 \mathrm{mg}, 0.5 \mathrm{mmol}, 1.0$ equiv) was added and the mixture was stirred for another $1 \mathrm{~h}$ to consume any traces of ring opened intermediate 2 . The mixture was then concentrated on a rotary evaporator and transferred to a separation funnel. DCM (50 $\mathrm{mL})$ was added, and the solution was washed with brine $(30 \mathrm{~mL})$. The organic phase was concentrated and purified with automated flash column chromatography using a $100 \mathrm{~g}$ SNAP cartridge unless otherwise specified.

Bulk Scale Preparation of 5j. Thiazolino fused 2-pyridone $\mathbf{1 j}$ (1.27 $\mathrm{g}, 2.57 \mathrm{mmol}, 1.0$ equiv) and cesium carbonate $(1.67 \mathrm{~g}, 5.14 \mathrm{mmol}$, 2.0 equiv) were weighed in an oven-dried $10-20 \mathrm{~mL}$ microwave vial and flushed with nitrogen. Dried THF $(7 \mathrm{~mL})$ and propargyl bromide ( $1.16 \mathrm{~mL}, 10.8 \mathrm{mmol}, 4.2$ equiv) were added to it, and the resulting mixture was stirred at $60{ }^{\circ} \mathrm{C}$. The mixture was then concentrated on a rotary evaporator and transferred to a separation funnel. DCM (50 $\mathrm{mL})$ was added, and the solution was washed with brine $(30 \mathrm{~mL})$. The organic phase was concentrated and purified with automated flash column chromatography using a $100 \mathrm{~g}$ SNAP cartridge to give $700 \mathrm{mg}$ of $\mathbf{5 j}$ in $51 \%$ yield.

Methyl 2-(5-Cyclopropyl-2-oxo-6-(prop-2-yn-1-ylthio)pyridin$1(2 \mathrm{H})$-yl)acrylate $(\mathbf{2 h})$. The compound was prepared following the general procedure, using propargyl bromide $(225 \mu \mathrm{L}, 2.10 \mathrm{mmol}, 4.2$ equiv). The crude product was purified with automated flash column chromatography (50 g Sfär cartridge, $10-80 \%$ EtOAc in heptane) to give pure $2 \mathrm{~h}$ as a light brown syrup ( $30 \mathrm{mg}, 0.10 \mathrm{mmol}, 20 \%)$ and $\mathbf{5 a}$ as a light brown powder $(65 \mathrm{mg}, 0.22 \mathrm{mmol}, 44 \%)$. IR (KBr): $\nu 3437$, $3288,3082,3002,2953,1733,1666,1590,1498,1438,1364,1327$, 1303, 1252, 1200, 1171, $1087 \mathrm{~cm}^{-1} .{ }^{1} \mathrm{H}$ NMR $\left(400 \mathrm{MHz}, \mathrm{CDCl}_{3}\right) \delta$ $6.88(\mathrm{~d}, J=9.7 \mathrm{~Hz}, 1 \mathrm{H}), 6.76(\mathrm{~s}, 1 \mathrm{H}), 6.67-6.57(\mathrm{~m}, 1 \mathrm{H}), 5.88(\mathrm{~s}$, $1 \mathrm{H}), 3.81(\mathrm{~s}, 3 \mathrm{H}), 3.49(\mathrm{dd}, J=16.0,2.6 \mathrm{~Hz}, 1 \mathrm{H}), 3.39(\mathrm{dd}, J=16.0$, $2.6 \mathrm{~Hz}, 1 \mathrm{H}), 2.45-2.38(\mathrm{~m}, 1 \mathrm{H}), 2.45-2.35(\mathrm{~m}, 1 \mathrm{H}), 1.10-0.90(\mathrm{~m}$, $2 \mathrm{H}), 0.68-0.65(\mathrm{~m}, 2 \mathrm{H}) .{ }^{13} \mathrm{C}\left\{{ }^{1} \mathrm{H}\right\}$ NMR $\left(100 \mathrm{MHz}, \mathrm{CDCl}_{3}\right) \delta 163.4$, 162.1, 137.2, 136.9, 136.1, 128.9, 127.7, 123.2, 77.8, 77.4, 77.1, 76.8, 73.3, 53.0, 24.8, 12.9, 8.26, 8.20. HRMS (ESI-TOF) $m / z:[\mathrm{M}+\mathrm{H}]^{+}$ calcd for $\mathrm{C}_{15} \mathrm{H}_{16} \mathrm{NO}_{3} \mathrm{~S}^{+} 290.0845$; observed 290.0848 .

Methyl 4-Cyclopropyl-2-methylene-7-oxo-2,2a-dihydro-7Hcyclobuta[4,5]thiazolo[3,2-a]pyridine-8a(1H)-carboxylate (5a). 5a was prepared following the procedure described above. Brown powder, $100 \mathrm{mg}$, 69\%. IR (KBr): $\nu$ 3437, 3083, 2349, 1744, 1657, 1585, 1498, 1436, $1303 \mathrm{~cm}^{-1} .{ }^{1} \mathrm{H}$ NMR $\left(400 \mathrm{MHz}, \mathrm{CDCl}_{3}\right) \delta 7.10$ $(\mathrm{d}, J=9.2 \mathrm{~Hz}, 1 \mathrm{H}), 6.22(\mathrm{~d}, J=9.2 \mathrm{~Hz}, 1 \mathrm{H}), 5.31-5.24(\mathrm{~m}, 1 \mathrm{H})$, $5.20-5.13(\mathrm{~m}, 1 \mathrm{H}), 4.80(\mathrm{q}, J=2.5 \mathrm{~Hz}, 1 \mathrm{H}), 4.01(\mathrm{dt}, J=17.3,2.9$ $\mathrm{Hz}, 1 \mathrm{H}), 3.11(\mathrm{dq}, J=17.3,2.5 \mathrm{~Hz}, 1 \mathrm{H}), 1.60-1.51(\mathrm{~m}, 1 \mathrm{H}), 0.89-$ $0.80(\mathrm{~m}, 2 \mathrm{H}), 0.62-0.53(\mathrm{~m}, 2 \mathrm{H}) .{ }^{13} \mathrm{C}$ NMR $\left(100 \mathrm{MHz}, \mathrm{CDCl}_{3}\right) \delta$ 168.12 , 161.11, 149.29, 145.15, 141.72, 115.25, 114.69, 113.13, 74.33, $53.41,51.60,41.17,12.49,6.54,6.17$. HRMS (ESI-TOF) $\mathrm{m} / z$ : $[\mathrm{M}+$ $\mathrm{H}]^{+}$calcd for $\mathrm{C}_{15} \mathrm{H}_{16} \mathrm{NO}_{3} \mathrm{~S}^{+} 290.0845$; observed 290.0846 .

Methyl 2-(6-(But-2-yn-1-ylthio)-5-cyclopropyl-2-oxopyridin1(2H)-yl)acrylate (2i). The compound was prepared from 1a (126 $\mathrm{mg}, 0.5 \mathrm{mmol}$ ) following the general procedure using 1-bromo-2butyne $(252 \mu \mathrm{L}, 2.1 \mathrm{mmol})$. After $23 \mathrm{~h}$, the ring opening reaction was complete. To be consistent with the preparation of $\mathbf{3 a}$ and to check if cycloaddition occurred, additional cesium carbonate $(163 \mathrm{mg}, 0.5$ mmol) was added. TLC showed no further reaction after $1 \mathrm{~h}$. The mixture was then concentrated on a rotary evaporator and transferred to a separation funnel. DCM $(50 \mathrm{~mL})$ was added, and the solution was washed with brine $(30 \mathrm{~mL})$. The organic layer was concentrated on a rotary evaporator and purified by automated flash column chromatography (50 g Sfär cartridge, $10-80 \%$ EtOAc in heptane) to give pure $2 \mathbf{i}$ as a light brown syrup ( $73 \mathrm{mg}, 0.24 \mathrm{mmol}, 48 \%$ ). IR $(\mathrm{KBr}): \nu$ 3443, 3081, 3001, 2952, 2851, 2234, 1734, 1669, 1592, $1498,1437,1363,1326,1303,1250,1200,1171,1086,1037 \mathrm{~cm}^{-1}$. ${ }^{1} \mathrm{H}$ NMR $\left[600 \mathrm{MHz},\left(\mathrm{CD}_{3}\right)_{2} \mathrm{SO}\right] \delta 7.04(\mathrm{~d}, J=9.6 \mathrm{~Hz}, 1 \mathrm{H}), 6.65(\mathrm{~s}$, $1 \mathrm{H}), 6.50(\mathrm{~d}, J=9.6 \mathrm{~Hz}, 1 \mathrm{H}), 6.04(\mathrm{~s}, 1 \mathrm{H}), 3.73(\mathrm{~s}, 3 \mathrm{H}), 3.59(\mathrm{dq}, J=$ $15.8,2.2 \mathrm{~Hz}, 1 \mathrm{H}), 3.43(\mathrm{dq}, J=15.8,2.3 \mathrm{~Hz}, 1 \mathrm{H}), 2.42-2.37(\mathrm{~m}$, $1 \mathrm{H}), 1.76(\mathrm{t}, J=2.6 \mathrm{~Hz}, 3 \mathrm{H}), 0.95-0.91(\mathrm{~m}, 2 \mathrm{H}), 0.73-0.61(\mathrm{~m}$, $2 \mathrm{H}) .{ }^{13} \mathrm{C}\left\{{ }^{1} \mathrm{H}\right\}$ NMR $\left[151 \mathrm{MHz},\left(\mathrm{CD}_{3}\right)_{2} \mathrm{SO}\right] \delta 163.1,160.9,136.9$, $136.8,135.5,128.0,127.7,122.3,81.2,73.6,52.7,24.9,12.5,7.44$, 7.42, 3.2. HRMS (ESI-TOF) $m / z$ : $[\mathrm{M}+\mathrm{H}]^{+}$calcd for $\mathrm{C}_{16} \mathrm{H}_{18} \mathrm{NO}_{3} \mathrm{~S}^{+}$ 304.1002; observed 304.1002. 
Methyl 2-Methylene-7-oxo-2,2a-dihydro-7H-cyclobuta[4,5]thiazolo[3,2-a]pyridine-8a(1H)-carboxylate $(\mathbf{5 b})$. $\mathbf{5 b}$ was prepared following the general procedure described above. Brown syrup, $60 \mathrm{mg}$, $48 \%$. IR (KBr): $\nu 1742,1639,1570,1511,1302,1221 \mathrm{~cm}^{-1} .{ }^{1} \mathrm{H}$ NMR $\left[600 \mathrm{MHz},\left(\mathrm{CD}_{3}\right)_{2} \mathrm{SO}\right] \delta 7.43(\mathrm{dd}, J=9.0,7.2 \mathrm{~Hz}, 1 \mathrm{H}), 6.31(\mathrm{dd}, J=$ $7.2,1.0 \mathrm{~Hz}, 1 \mathrm{H}), 6.13(\mathrm{dd}, J=9.0,1.0 \mathrm{~Hz}, 1 \mathrm{H}), 5.34-5.25(\mathrm{~m}, 2 \mathrm{H})$, $5.19(\mathrm{t}, J=2.0 \mathrm{~Hz}, 1 \mathrm{H}), 3.81-3.77(\mathrm{~m}, 1 \mathrm{H}), 3.69(\mathrm{~s}, 3 \mathrm{H}), 2.94(\mathrm{dd}, J$ $=17.1,2.5 \mathrm{~Hz}, 1 \mathrm{H}) .{ }^{13} \mathrm{C}\left\{{ }^{1} \mathrm{H}\right\} \mathrm{NMR}\left[151 \mathrm{MHz},\left(\mathrm{CD}_{3}\right)_{2} \mathrm{SO}\right] \delta 167.3$, 160.4, 150.0, 145.2, 141.8, 114.2, 112.8, 100.4, 72.8, 53.0, 51.1, 39.8, 39.6, 39.5, 39.3, 39.2. HRMS (ESI-TOF) $\mathrm{m} / z$ : $[\mathrm{M}+\mathrm{H}]^{+}$calcd for $\mathrm{C}_{12} \mathrm{H}_{12} \mathrm{NO}_{3} \mathrm{~S}^{+}$250.0532; observed 250.0534 .

Methyl 4-Methoxy-2-methylene-7-oxo-2,2a-dihydro-7Hcyclobuta[4,5]thiazolo[3,2-a]pyridine-8a(1H)-carboxylate (5c). 5c was prepared following the general procedure described above. Brown syrup, $80 \mathrm{mg}$, 57\%. IR (KBr): $\nu$ 3436, 2952, 2837, 1744, 1663, 1579, $1503,1453,1435,1411,1353,1303,1268,1218,1178,1150,1088$, $1051 \mathrm{~cm}^{-1} .{ }^{1} \mathrm{H}$ NMR $\left[600 \mathrm{MHz},\left(\mathrm{CD}_{3}\right)_{2} \mathrm{SO}\right] \delta 7.60(\mathrm{~d}, J=9.7 \mathrm{~Hz}$, $1 \mathrm{H}), 6.13(\mathrm{~d}, J=9.7 \mathrm{~Hz}, 1 \mathrm{H}), 5.39-5.26(\mathrm{~m}, 2 \mathrm{H}), 5.24-5.15(\mathrm{~m}$, $1 \mathrm{H}), 3.77(\mathrm{dt}, J=17.2,2.8 \mathrm{~Hz}, 1 \mathrm{H}), 3.71(\mathrm{~s}, 3 \mathrm{H}), 3.69(\mathrm{~s}, 3 \mathrm{H}), 2.97-$ $2.92(\mathrm{~m}, 1 \mathrm{H}) .{ }^{13} \mathrm{C}\left\{{ }^{1} \mathrm{H}\right\}$ NMR $\left[151 \mathrm{MHz},\left(\mathrm{CD}_{3}\right)_{2} \mathrm{SO}\right] \delta 167.2,158.4$, 145.2, 137.4, 136.4, 133.6, 114.4, 112.9, 73.5, 58.5, 53.0, 51.8. HRMS (ESI-TOF) $m / z:[\mathrm{M}+\mathrm{H}]^{+}$calcd for $\mathrm{C}_{13} \mathrm{H}_{14} \mathrm{~N}_{4} \mathrm{OS}^{+}$280.0638; observed 280.0640 .

Methyl 4-Cyclopropyl-6-iodo-2-methylene-7-oxo-2,2a-dihydro$7 \mathrm{H}$-cyclobuta[4,5]thiazolo[3,2-a]pyridine-8a(1H)-carboxylate $(5 d)$. $\mathbf{5 d}$ was prepared following the general procedure described above. Brown syrup, $120 \mathrm{mg}$, 58\%. IR (KBr): $\nu$ 1745, 1649, 1578, 1481, 1433, 1329, 1301, 1244, 1218, 1175, 1147, 1089, $1025 \mathrm{~cm}^{-1} \cdot{ }^{1} \mathrm{H}$ NMR $\left[600 \mathrm{MHz},\left(\mathrm{CD}_{3}\right)_{2} \mathrm{SO}\right] \delta 7.72(\mathrm{~s}, 1 \mathrm{H}), 5.32(\mathrm{t}, J=2.5 \mathrm{~Hz}, 2 \mathrm{H})$, 5.24-5.14 (m, 1H), $3.79(\mathrm{dt}, J=17.3,2.6 \mathrm{~Hz}, 1 \mathrm{H}), 3.70(\mathrm{~s}, 3 \mathrm{H})$, $1.52-1.50(\mathrm{~m}, 1 \mathrm{H}), 0.89-0.73(\mathrm{~m}, 2 \mathrm{H}), 0.63-0.59(\mathrm{~m}, 2 \mathrm{H})$. ${ }^{13} \mathrm{C}\left\{{ }^{1} \mathrm{H}\right\}$ NMR $\left[151 \mathrm{MHz},\left(\mathrm{CD}_{3}\right)_{2} \mathrm{SO}\right] \delta 167.0,157.2,150.4,148.9$, $144.9,115.3,113.0,84.6,74.2,53.1,51.4,11.9,6.4,6.1$. HRMS (ESITOF) $m / z:[\mathrm{M}+\mathrm{H}]^{+}$calcd for $\mathrm{C}_{15} \mathrm{H}_{15} \mathrm{INO}_{3} \mathrm{~S}^{+}$415.9812; observed 415.9814.

Methyl 4-Cyclopropyl-2-methylene-7-oxo-6-(p-tolyl)-2,2a-dihydro-7H-cyclobuta[4,5]thiazolo[3,2-a]pyridine-8a(1H)-carboxylate (5e). Colorless syrup, $146 \mathrm{mg}$, 77\%. IR (KBr): L 3734, 3087, 3018, 2954, 2917, 1739, 1682, 1639, 1593, 1527, 1507, 1427, 1375, 1341, $1298,1269,1242,1217,1196,1183,1171,1111,1089,1046,1036$, $1023 \mathrm{~cm}^{-1} .{ }^{1} \mathrm{H}$ NMR $\left[600 \mathrm{MHz},\left(\mathrm{CD}_{3}\right)_{2} \mathrm{SO}\right] \delta 7.57(\mathrm{~d}, J=8.0 \mathrm{~Hz}$, $2 \mathrm{H}), 7.29(\mathrm{~s}, 1 \mathrm{H}), 7.16(\mathrm{~d}, J=7.9 \mathrm{~Hz}, 2 \mathrm{H}), 5.35-5.34(\mathrm{~m}, 2 \mathrm{H}), 5.20$ $(\mathrm{s}, 1 \mathrm{H}), 3.83(\mathrm{dt}, J=17.2,2.6 \mathrm{~Hz}, 1 \mathrm{H}), 3.70(\mathrm{~s}, 3 \mathrm{H}), 3.02(\mathrm{dd}, J=$ $17.2,2.6 \mathrm{~Hz}, 1 \mathrm{H}), 2.31(\mathrm{~s}, 3 \mathrm{H}), 1.59-1.56(\mathrm{~m}, 1 \mathrm{H}), 0.88-0.80(\mathrm{~m}$, $2 \mathrm{H}), 0.76-0.63(\mathrm{~m}, 2 \mathrm{H}) .{ }^{13} \mathrm{C}\left\{{ }^{1} \mathrm{H}\right\} \operatorname{NMR}\left[151 \mathrm{MHz},\left(\mathrm{CD}_{3}\right)_{2} \mathrm{SO}\right] \delta$ $167.5,158.6,147.3,145.4,138.1,136.4,132.9,128.4,128.0,124.7$, $113.6,112.8,73.7,52.9,51.2,40.6,39.9,39.8,39.6,39.5,39.3,39.2$, 39.1, 20.7, 12.3, 6.3, 6.1. HRMS (ESI-TOF) $\mathrm{m} / z$ : $[\mathrm{M}+\mathrm{H}]^{+}$calcd for $\mathrm{C}_{22} \mathrm{H}_{22} \mathrm{NO}_{3} \mathrm{~S}^{+}$380.1315; observed 380.1319.

Methyl 4-Cyclopropyl-6-(4-methoxyphenyl)-2-methylene-7-oxo2,2a-dihydro-7H-cyclobuta[4,5]thiazolo[3,2-a]pyridine-8a(1H)-carboxylate (5f). Colorless syrup, $146 \mathrm{mg}, 74 \%$. IR (KBr): $\nu 2241,1747$, 1691,1632 , 1604, 1585, 1519, 1453, 1385, 1342, 1305, 1290, 1249, $1216,1179,1141,1114,1089,1058,1029 \mathrm{~cm}^{-1}$. ${ }^{1} \mathrm{H}$ NMR $[600 \mathrm{MHz}$, $\left.\left(\mathrm{CD}_{3}\right)_{2} \mathrm{SO}\right] \delta 7.66-7.59(\mathrm{~m}, 2 \mathrm{H}), 7.27(\mathrm{~s}, 1 \mathrm{H}), 6.97-6.87(\mathrm{~m}, 2 \mathrm{H})$, $5.34(\mathrm{t}, J=2.5 \mathrm{~Hz}, 2 \mathrm{H}), 5.21(\mathrm{t}, J=2.0 \mathrm{~Hz}, 1 \mathrm{H}), 3.83(\mathrm{dt}, J=17.6$, $2.7 \mathrm{~Hz}, 1 \mathrm{H}), 3.77(\mathrm{~s}, 3 \mathrm{H}), 3.70(\mathrm{~s}, 3 \mathrm{H}), 3.08-2.97(\mathrm{~m}, 1 \mathrm{H}), 1.60-$ $1.55(\mathrm{~m}, 1 \mathrm{H}), 0.88-0.79(\mathrm{~m}, 2 \mathrm{H}), 0.74-0.62(\mathrm{~m}, 2 \mathrm{H}) .{ }^{13} \mathrm{C}\left\{{ }^{1} \mathrm{H}\right\}$ NMR [151 MHz, $\left.\left(\mathrm{CD}_{3}\right)_{2} \mathrm{SO}\right] \delta 167.5,158.7,158.5,146.7,145.5$, 137.5, 129.3, 128.1, 124.5, 113.6, 113.3, 112.8, 73.7, 55.1, 52.9, 51.2, $12.3,6.3,6.1$. HRMS (ESI-TOF) $m / z:[\mathrm{M}+\mathrm{H}]^{+}$calcd for $\mathrm{C}_{22} \mathrm{H}_{22} \mathrm{NO}_{4} \mathrm{~S}^{+}$396.1263; observed 396.1263.

Methyl 4-Cyclopropyl-2-methylene-6-(4-nitrophenyl)-7-oxo2,2a-dihydro-7H-cyclobuta[4,5]thiazolo[3,2-a]pyridine-8a(1H)-carboxylate (5g). Colorless syrup, $140 \mathrm{mg}, 68 \%$. IR (KBr): $\nu$ 3730, 1746, $1641,1590,1509,1434,1338,1308,1265,1242,1218,1172,1136$, $1107,1089,1055,1031 \mathrm{~cm}^{-1}$. ${ }^{1} \mathrm{H}$ NMR $\left[600 \mathrm{MHz},\left(\mathrm{CD}_{3}\right)_{2} \mathrm{SO}\right] \delta$ $8.26-8.16(\mathrm{~m}, 2 \mathrm{H}), 8.10-8.00(\mathrm{~m}, 2 \mathrm{H}), 7.56(\mathrm{~s}, 1 \mathrm{H}), 5.41(\mathrm{q}, J=2.5$ $\mathrm{Hz}, 1 \mathrm{H}), 5.37(\mathrm{q}, J=2.8 \mathrm{~Hz}, 1 \mathrm{H}), 5.22(\mathrm{~s}, 1 \mathrm{H}), 3.85(\mathrm{dt}, J=17.4,2.8$ $\mathrm{Hz}, 1 \mathrm{H}), 3.72(\mathrm{~s}, 3 \mathrm{H}), 3.10(\mathrm{dq}, J=17.4,2.6 \mathrm{~Hz}, 1 \mathrm{H}), 1.62-1.59(\mathrm{~m}$, $1 \mathrm{H}), 0.88-0.86(\mathrm{~m}, 2 \mathrm{H}), 0.78-0.65(\mathrm{~m}, 2 \mathrm{H}) .{ }^{13} \mathrm{C}\left\{{ }^{1} \mathrm{H}\right\} \operatorname{NMR}[151$
$\left.\mathrm{MHz},\left(\mathrm{CD}_{3}\right)_{2} \mathrm{SO}\right] \delta 167.2,158.2,150.9,145.9,145.1,142.7,140.2$, $128.9,123.0,121.7,114.1,113.1,73.9,53.1,51.3,12.3,6.4,6.2$. HRMS (ESI-TOF) $m / z:[\mathrm{M}+\mathrm{H}]^{+}$calcd for $\mathrm{C}_{21} \mathrm{H}_{19} \mathrm{~N}_{2} \mathrm{O}_{5} \mathrm{~S}^{+}$ 411.1009; observed 411.1013.

Methyl 4-Cyclopropyl-2-methylene-7-oxo-6-(thiophen-3-yl)2,2a-dihydro-7H-cyclobuta[4,5]thiazolo[3,2-a]pyridine-8a(1H)-carboxylate (5h). Colorless syrup, $120 \mathrm{mg}, 65 \%$. IR (KBr): $\nu$ 3556, 2933, $1739,1676,1636,1585,1526,1502,1449,1425,1400,1374,1351$, $1305,1271,1248,1231,1216,1194,1165,1126,1107,1084,1056$, $1031 \mathrm{~cm}^{-1} .{ }^{1} \mathrm{H}$ NMR $\left[600 \mathrm{MHz},\left(\mathrm{CD}_{3}\right)_{2} \mathrm{SO}\right] \delta 8.17(\mathrm{dd}, J=3.1,1.3$ $\mathrm{Hz}, 1 \mathrm{H}), 7.66(\mathrm{dd}, J=5.2,1.3 \mathrm{~Hz}, 1 \mathrm{H}), 7.56(\mathrm{~s}, 1 \mathrm{H}), 7.53(\mathrm{dd}, J=$ 5.1, $3.1 \mathrm{~Hz}, 1 \mathrm{H}), 5.36-5.34(\mathrm{~m}, 2 \mathrm{H}), 5.22-5.18(\mathrm{~m}, 1 \mathrm{H}), 3.85(\mathrm{dt}, J$ $=17.3,2.8 \mathrm{~Hz}, 1 \mathrm{H}), 3.71(\mathrm{~s}, 3 \mathrm{H}), 3.03(\mathrm{dq}, J=17.2,2.6 \mathrm{~Hz}, 1 \mathrm{H})$, $1.61-1.56(\mathrm{~m}, 1 \mathrm{H}), 0.89-0.84(\mathrm{~m}, 2 \mathrm{H}), 0.78-0.63(\mathrm{~m}, 2 \mathrm{H})$. ${ }^{13} \mathrm{C}\left\{{ }^{1} \mathrm{H}\right\}$ NMR $\left[151 \mathrm{MHz},\left(\mathrm{CD}_{3}\right)_{2} \mathrm{SO}\right] \delta 167.4,158.2,146.8,145.3$, 136.6, 135.8, 126.9, 125.1, 123.1, 119.9, 113.5, 112.8, 73.7, 53.0, 51.1, 40.6, 40.0, 39.9, 39.8, 39.6, 39.5, 39.3, 39.2, 39.1, 12.4, 6.4, 6.2. HRMS (ESI-TOF) $m / z:[\mathrm{M}+\mathrm{H}]^{+}$calcd for $\mathrm{C}_{19} \mathrm{H}_{18} \mathrm{FNO}_{3} \mathrm{~S}_{2}^{+} 372.0723$; observed 372.0724 .

Methyl 4-Cyclopropyl-2-methylene-5-(naphthalen-1-ylmethyl)7-oxo-2,2a-dihydro-7H-cyclobuta[4,5]thiazolo[3,2-a]pyridine$8 a(1 \mathrm{H})$-carboxylate (5i). The compound was prepared following the general procedure, but after addition of $\mathrm{Cs}_{2} \mathrm{CO}_{3}(325 \mathrm{mg}, 1 \mathrm{mmol})$, the reaction mixture was stirred for $2 \mathrm{~h}$. White powder, $105 \mathrm{mg}, 49 \%$. IR $(\mathrm{KBr}): \nu 3087,3004,2952,1745,1652,1577,1488,1428,1398$, $1363,1331,1302,1290,1264,1216,1160,1092,1067,1030 \mathrm{~cm}^{-1}$. ${ }^{1} \mathrm{H}$ NMR $\left[600 \mathrm{MHz},\left(\mathrm{CD}_{3}\right)_{2} \mathrm{SO}\right] \delta 8.00-7.94(\mathrm{~m}, 1 \mathrm{H}), 7.94-7.85$ $(\mathrm{m}, 2 \mathrm{H}), 7.56-7.47(\mathrm{~m}, 3 \mathrm{H}), 7.41(\mathrm{dd}, J=7.0,1.2 \mathrm{~Hz}, 1 \mathrm{H}), 5.32-$ $5.31(\mathrm{~m}, 1 \mathrm{H}), 5.24-5.22(\mathrm{~m}, 1 \mathrm{H}), 5.20-5.17(\mathrm{~m}, 1 \mathrm{H}) 5.17-5.16(\mathrm{~m}$, $1 \mathrm{H}), 4.51-4.42(\mathrm{~m}, 2 \mathrm{H}), 3.73(\mathrm{dt}, J=17.1,2.9 \mathrm{~Hz}, 1 \mathrm{H}), 3.62(\mathrm{~d}, J=$ $18.4 \mathrm{~Hz}, 3 \mathrm{H}), 2.85(\mathrm{dq} J=17.1,2.6 \mathrm{~Hz}, 1 \mathrm{H}), 1.80-1.74(\mathrm{~m}, 1 \mathrm{H})$, 0.99-0.90 (m, 2H), 0.82-0.69 (m, 2H). ${ }^{13} \mathrm{C}\left\{{ }^{1} \mathrm{H}\right\}$ NMR [151 MHz, $\left.\left(\mathrm{CD}_{3}\right)_{2} \mathrm{SO}\right] \delta 167.5,159.2,157.6,149.6,145.4,134.2,133.4,131.6$, $128.6,127.8,127.4,126.4,125.8,125.7,124.1,113.4,112.7,112.4$, 72.4, 52.9, 51.0, 40.4, 40.0, 39.9, 39.8, 39.6, 39.5, 39.3, 39.2, 39.1, 35.2 , 10.7, 7.7, 7.4. HRMS (ESI-TOF) $\mathrm{m} / \mathrm{z}:[\mathrm{M}+\mathrm{H}]^{+}$calcd for $\mathrm{C}_{26} \mathrm{H}_{24} \mathrm{NO}_{3} \mathrm{~S}^{+}$430.1471; observed 430.1467 .

Methyl 2-Methylene-5-(naphthalen-1-ylmethyl)-7-oxo-4-(3(trifluoromethyl)phenyl)-2,2a-dihydro-7H-cyclobuta[4,5] thiazolo[3,2-a]pyridine-8a(1H)-carboxylate (5j). Yellow powder, $105 \mathrm{mg}$, 49\%. IR (KBr): $\nu$ 3061, 2953, 1746, 1656, 1579, 1482, 1435, 1330, $1292,1267,1218,1165,1125,1094,1072,1046,1018 \mathrm{~cm}^{-1} .{ }^{1} \mathrm{H}$ NMR [600 MHz, $\left.\left(\mathrm{CD}_{3}\right)_{2} \mathrm{SO}\right] \delta 7.93-7.89(\mathrm{~m}, 1 \mathrm{H}), 7.82(\mathrm{~d}, J=8.3$ $\mathrm{Hz}, 1 \mathrm{H}), 7.78-7.76(\mathrm{~m}, 2 \mathrm{H}), 7.72(\mathrm{dd}, J=15.6,7.3 \mathrm{~Hz}, 2 \mathrm{H}), 7.66$ $(\mathrm{dd}, J=14.2,6.5 \mathrm{~Hz}, 1 \mathrm{H}), 7.48(\mathrm{p}, J=6.9 \mathrm{~Hz}, 2 \mathrm{H}), 7.43(\mathrm{dd}, J=8.2$, $7.0 \mathrm{~Hz}, 1 \mathrm{H}), 7.28-7.24(\mathrm{~m}, 1 \mathrm{H}), 5.57(\mathrm{~d}, J=13.6 \mathrm{~Hz}, 1 \mathrm{H}), 5.32-$ $5.14(\mathrm{~m}, 3 \mathrm{H}), 4.02(\mathrm{qd}, J=17.0,10.0 \mathrm{~Hz}, 2 \mathrm{H}), 3.78(\mathrm{dt}, J=17.2,2.8$ $\mathrm{Hz}, 1 \mathrm{H}), 3.70(\mathrm{~s}, 3 \mathrm{H}), 3.05-2.89(\mathrm{~m}, 1 \mathrm{H}) .{ }^{13} \mathrm{C}\left\{{ }^{1} \mathrm{H}\right\}$ NMR $[151$ $\left.\mathrm{MHz},\left(\mathrm{CD}_{3}\right)_{2} \mathrm{SO}\right] \delta 167.2,159.3,154.5,154.5,149.8,145.0,136.8$, $136.7,134.4,134.3,133.6,133.3,131.2,131.2,130.1,130.0,128.5$, $127.7,127.6,127.4,126.7,126.2$, 125.7, 125.5, 125.1, 123.7, 123.7, $114.1,114.0,113.5,113.1,79.1,78.9,78.7,73.5,73.4,53.1,51.2,40.5$, 40.5, 40.0, 39.9, 39.8, 39.6, 39.5, 39.3, 39.2, 39.1, 35.7, 31.2. ${ }^{19} \mathrm{~F}$ NMR $\left[400 \mathrm{MHz},\left(\mathrm{CD}_{3}\right)_{2} \mathrm{SO}\right] \delta-61.25$. HRMS (ESI-TOF) $\mathrm{m} / z:[\mathrm{M}+\mathrm{H}]^{+}$ calcd for $\mathrm{C}_{30} \mathrm{H}_{23} \mathrm{~F}_{3} \mathrm{NO}_{3} \mathrm{~S}^{+}$534.1345; observed 534.1348.

Methyl 4-(Dimethylamino)-2-methylene-5-((4-methylnaphthalen-1-yl)methyl)-7-oxo-2,2a-dihydro-7H-cyclobuta[4,5] thiazolo[3,2-a]pyridine-8a(1H)-carboxylate $(\mathbf{5 k})$. Colorless powder, $130 \mathrm{mg}$, $58 \%$. IR (KBr): $\nu$ 3442, 2930, 2860, 2828, 2785, 1745, 1653, 1573, $1481,1435,1392,1338,1298,1248,1216,1171,1149,1088,1063$ $\mathrm{cm}^{-1} .{ }^{1} \mathrm{H}$ NMR $\left[400 \mathrm{MHz},\left(\mathrm{CD}_{3}\right)_{2} \mathrm{SO}\right] \delta 8.10-8.03(\mathrm{~m}, 1 \mathrm{H}), 7.94-$ $7.86(\mathrm{~m}, 1 \mathrm{H}), 7.62-7.52(\mathrm{~m}, 2 \mathrm{H}), 7.39-7.27(\mathrm{~m}, 2 \mathrm{H}), 5.33-5.31$ $(\mathrm{m}, 1 \mathrm{H}), 5.28-5.21(\mathrm{~m}, 2 \mathrm{H}), 5.19-5.17(\mathrm{~m}, 1 \mathrm{H}), 4.29(\mathrm{~s}, 2 \mathrm{H})$, 3.75-3.74 (m, 1H), $3.65(\mathrm{~s}, 3 \mathrm{H}), 2.87-2.85(\mathrm{~m}, 1 \mathrm{H}), 2.75(\mathrm{~s}, 5 \mathrm{H})$, $2.68-2.63(\mathrm{~m}, 3 \mathrm{H}) .{ }^{13} \mathrm{C}\left\{{ }^{1} \mathrm{H}\right\}$ NMR $\left[100 \mathrm{MHz},\left(\mathrm{CD}_{3}\right)_{2} \mathrm{SO}\right] \delta 167.4$, $158.8,157.4,148.5,145.4,133.2$, 132.5, 132.4, 131.5, 127.5, 126.2, 126.0, 125.7, 125.4, 124.8, 124.5, 113.3, 112.8, 72.7, 52.9, 51.3, 33.7, 19.0. HRMS (ESI-TOF) $m / z$ : $[\mathrm{M}+\mathrm{H}]^{+}$calcd for $\mathrm{C}_{26} \mathrm{H}_{27} \mathrm{~N}_{2} \mathrm{O}_{3} \mathrm{~S}^{+}$ 447.1737; observed 447.1747.

Methyl 2-Methylene-5-(naphthalen-1-ylmethyl)-7-oxo-2,2a-dihydro-7H-cyclobuta[4,5] thiazolo[3,2-a]pyridine-8a(1H)-carboxy- 
late (5I). White powder, $81 \mathrm{mg}, 41 \%$. IR (KBr): $\nu 2952,1743,1656$, $1573,1506,1435,1396,1306,1222,1156,1090,1068,1018 \mathrm{~cm}^{-1}$. ${ }^{1} \mathrm{H}$ NMR [ $\left.400 \mathrm{MHz},\left(\mathrm{CD}_{3}\right)_{2} \mathrm{SO}\right] \delta 8.08-8.03(\mathrm{~m}, 1 \mathrm{H}), 7.95(\mathrm{dd}, J=$ $7.3,2.1 \mathrm{~Hz}, 1 \mathrm{H}), 7.90-7.83(\mathrm{~m}, 1 \mathrm{H}), 7.59-7.46(\mathrm{~m}, 4 \mathrm{H}), 6.23(\mathrm{~d}, J=$ $1.4 \mathrm{~Hz}, 1 \mathrm{H}), 5.88(\mathrm{~d}, J=1.4 \mathrm{~Hz}, 1 \mathrm{H}), 5.26(\mathrm{q}, J=2.6 \mathrm{~Hz}, 2 \mathrm{H}), 5.14$ $(\mathrm{q}, J=2.0 \mathrm{~Hz}, 1 \mathrm{H}), 4.25(\mathrm{~s}, 2 \mathrm{H}), 3.73(\mathrm{dt}, J=17.1,2.7 \mathrm{~Hz}, 1 \mathrm{H}), 3.65$ $(\mathrm{s}, 3 \mathrm{H}), 2.88(\mathrm{dq}, J=17.2,2.6 \mathrm{~Hz}, 1 \mathrm{H}) .{ }^{13} \mathrm{C}\left\{{ }^{1} \mathrm{H}\right\}$ NMR $[100 \mathrm{MHz}$, $\left.\left(\mathrm{CD}_{3}\right)_{2} \mathrm{SO}\right] \delta 167.3,160.1,156.0,149.3,145.1,134.2,133.5,131.4$, $128.6,127.8,127.4,126.3,125.8,125.7,124.0,112.8,112.7,101.5$, 72.4, 52.9, 51.3, 37.4. HRMS (ESI-TOF) $m / z:[\mathrm{M}+\mathrm{H}]^{+}$calcd for $\mathrm{C}_{23} \mathrm{H}_{20} \mathrm{NO}_{3} \mathrm{~S}^{+}$390.1158; observed 390.1157.

(R)-1-Phenylethyl (8a)-4-Cyclopropyl-2-methylene-5-(naphthalen-1-ylmethyl)-7-oxo-2,2a-dihydro-7H-cyclobuta[4,5] thiazolo[3,2a]pyridine-8a(1H)-carboxylate (a Diastereomeric Mixture of $5 \mathrm{~m}$ ). Starting from $1 \mathrm{~m}(170 \mathrm{mg}, 0.353 \mathrm{mmol}, 1.0$ equiv), the product was synthesized following the general procedure and isolated in 33\% yield $(170 \mathrm{mg}) .5 \mathrm{~m}$ as white powder. IR (KBr): $\nu 1740,1654,1487,1285$, $1160,780 \mathrm{~cm}^{-1} ;{ }^{1} \mathrm{H}$ NMR $\left(400 \mathrm{MHz}, \mathrm{CDCl}_{3}\right) \delta 7.81-7.76(\mathrm{~m}, 2 \mathrm{H})$, 7.74-7.67 (m, 4H), 7.42-7.34 (m, 4H), 7.34-7.30 (m, 2H), 7.29$7.20(\mathrm{~m}, 3 \mathrm{H}), 7.20-7.13(\mathrm{~m}, 9 \mathrm{H}), 5.84(\mathrm{dq} J=13.0,6.5 \mathrm{~Hz}, 2 \mathrm{H})$, $5.66(\mathrm{~s}, 1 \mathrm{H}), 5.62(\mathrm{~s}, 1 \mathrm{H}), 5.23-5.15(\mathrm{~m}, 2 \mathrm{H}), 5.12-5.01(\mathrm{~m}, 2 \mathrm{H})$, $4.62-4.51(\mathrm{~m}, 2 \mathrm{H}), 4.42-4.26(\mathrm{~m}, 4 \mathrm{H}), 3.88(\mathrm{ddt}, J=17.2,8.9,2.8$ $\mathrm{Hz}, 2 \mathrm{H}), 3.03$ (tdd, $J=14.9,4.9,2.5 \mathrm{~Hz}, 2 \mathrm{H}), 1.62-1.52(\mathrm{~m}, 2 \mathrm{H})$, $1.51(\mathrm{~d}, J=6.6 \mathrm{~Hz}, 3 \mathrm{H}), 1.36(\mathrm{~d}, J=6.6 \mathrm{~Hz}, 3 \mathrm{H}), 0.93-0.81(\mathrm{~m}$, $4 \mathrm{H}), 0.69(\mathrm{~m}, J=7.2,5.5 \mathrm{~Hz}, 4 \mathrm{H}) ;{ }^{13} \mathrm{C}\left\{{ }^{1} \mathrm{H}\right\}$ NMR $[100 \mathrm{MHz}$, $\left.\mathrm{CDCl}_{3}\right] \delta 167.0,161.0,157.3,149.8,145.6,141.4,140.9,134.2$, 132.1, $129.0,128.6,128.0,127.8,127.6,126.3,126.0,125.8,125.7,123.9$, 115.7, 113.3, 112.8, 74.5, 51.4, 40.8, 36.4, 22.2, 11.2, 8.3, 7.9; HRMS (ESI-TOF) $m / z[\mathrm{M}+\mathrm{H}]^{+}$calcd for $\mathrm{C}_{33} \mathrm{H}_{30} \mathrm{NO}_{3} \mathrm{~S}^{+}$520.1946, found 520.1965.

General Procedure for Synthesis of $\mathbf{6 a}-\boldsymbol{c}$. Methyl ester $\mathbf{5 j - 1}$ was dissolved in THF, and $\mathrm{LiOH}$ (0.10 M, 4.5 equiv) was added. Upon completion, $\mathrm{HCl}$ ( $1.00 \mathrm{M}, 5.0$ equiv) was added. The mixture was stirred for $1 \mathrm{~min}$ and concentrated on a rotary evaporator. The residue was dissolved in EtOAc $(50 \mathrm{~mL})$ and washed with brine $(30 \mathrm{~mL})$. The organic phase was concentrated on a rotary evaporator, dissolved in DMSO, filtered, and purified with preparative HPLC.

4-Cyclopropyl-2-methylene-5-(naphthalen-1-ylmethyl)-7-oxo2,2a-dihydro-7H-cyclobuta[4,5]thiazolo[3,2-a]pyridine-8a(1H)-carboxylic Acid (6a). The reaction was performed on $39 \mathrm{mg}(0.09$ $\mathrm{mmol})$ of $5 \mathbf{i}$ in THF $(4 \mathrm{~mL})$ and showed completion in $6 \mathrm{~h}$. White powder, $20 \mathrm{mg}$, 53\%. IR (KBr): $\nu$ 3425, 3086, 1944, 1724, 1621, $1539,1509,1485,1416,1393,1359,1294,1272,1214,1180,1161$, $1025 \mathrm{~cm}^{-1} .{ }^{1} \mathrm{H}$ NMR $\left[600 \mathrm{MHz},\left(\mathrm{CD}_{3}\right)_{2} \mathrm{SO}\right] \delta 7.98-7.96(\mathrm{~m}, 1 \mathrm{H})$, 7.93-7.86 (m, 2H), 7.56-7.48 (m, 3H), 7.42-7.36 (m, 1H), 5.29$5.28(\mathrm{~m}, 1 \mathrm{H}), 5.18(\mathrm{~s}, 1 \mathrm{H}), 5.15-5.14(\mathrm{~m}, 2 \mathrm{H}), 4.46(\mathrm{~s}, 2 \mathrm{H}), 3.68$ $(\mathrm{dt}, J=16.9,2.7 \mathrm{~Hz}, 1 \mathrm{H}), 2.82(\mathrm{dq}, J=17.1,2.5 \mathrm{~Hz}, 1 \mathrm{H}), 1.79-1.74$ (m, $1 \mathrm{H}), 1.00-0.87(\mathrm{~m}, 2 \mathrm{H}), 0.78-0.73(\mathrm{~m}, 2 \mathrm{H}) .{ }^{13} \mathrm{C}\left\{{ }^{1} \mathrm{H}\right\} \mathrm{NMR}$ $\left[151 \mathrm{MHz},\left(\mathrm{CD}_{3}\right)_{2} \mathrm{SO}\right] \delta 168.5,159.3,157.3,149.8,145.9,134.3$, $133.4,131.6,128.6,127.8,127.3,126.4,125.8,125.7,124.1,113.5$, $112.3,112.1,72.9,51.0,35.2,10.7,7.6,7.5$. HRMS (ESI-TOF) $\mathrm{m} / z$ : $[\mathrm{M}+\mathrm{H}]^{+}$calcd for $\mathrm{C}_{25} \mathrm{H}_{22} \mathrm{NO}_{3} \mathrm{~S}^{+} 416.1315$; observed 416.1319 .

2-Methylene-5-(naphthalen-1-ylmethyl)-7-oxo-4-(3(trifluoromethyl)phenyl)-2,2a-dihydro-7H-cyclobuta[4,5]thiazolo[3,2-a]pyridine-8a(1H)-carboxylic Acid $(\mathbf{6 b})$. The reaction was performed on $48 \mathrm{mg}(0.09 \mathrm{mmol})$ of $\mathbf{5 j}$ in THF $(4 \mathrm{~mL})$ and showed completion in $6 \mathrm{~h}$. White powder, $10 \mathrm{mg}, 21 \%$. IR (KBr): $\nu 3446$, $1733,1638,1576,1483,1437,1397,1377,1335,1299,1269,1220$, $1166,1125,1094,1073,1046 \mathrm{~cm}^{-1} .{ }^{1} \mathrm{H}$ NMR $\left[600 \mathrm{MHz},\left(\mathrm{CD}_{3}\right)_{2} \mathrm{SO}\right]$ $\delta 7.91(\mathrm{dd}, J=7.4,1.9 \mathrm{~Hz}, 1 \mathrm{H}), 7.82(\mathrm{~d}, J=8.2 \mathrm{~Hz}, 1 \mathrm{H}), 7.80-7.74$ (m, 2H), 7.74-7.61 (m, 3H), 7.50-7.48 (m, 2H), 7.45-7.42 (m, $1 \mathrm{H}), 7.26(\mathrm{~d}, J=7.0 \mathrm{~Hz}, 1 \mathrm{H}), 5.54(\mathrm{~d}, J=6.0 \mathrm{~Hz}, 1 \mathrm{H}), 5.23(\mathrm{~s}, 1 \mathrm{H})$, $5.16(\mathrm{~s}, 1 \mathrm{H}), 5.13(\mathrm{~s}, 1 \mathrm{H}), 4.06-3.95(\mathrm{~m}, 2 \mathrm{H}), 3.73(\mathrm{dt}, J=17.1,2.9$ $\mathrm{Hz}, 1 \mathrm{H}), 2.95(\mathrm{dd}, J=16.9,3.3 \mathrm{~Hz}, 1 \mathrm{H}) .{ }^{13} \mathrm{C}\left\{{ }^{1} \mathrm{H}\right\}$ NMR $[151 \mathrm{MHz}$, $\left.\left(\mathrm{CD}_{3}\right)_{2} \mathrm{SO}\right] \delta 168.3,159.4,154.1,150.0,145.6,133.7,133.3,131.2$, $130.0,128.5,127.6,127.6,127.3,126.2,125.7,125.5,123.7,114.1$, 113.3, 112.7, 74.0, 51.3, 35.7. ${ }^{19} \mathrm{~F}$ NMR [ $\left.400 \mathrm{MHz},\left(\mathrm{CD}_{3}\right)_{2} \mathrm{SO}\right] \delta$ -61.24. HRMS (ESI-TOF) $\mathrm{m} / z$ : $[\mathrm{M}+\mathrm{H}]^{+}$calcd for $\mathrm{C}_{29} \mathrm{H}_{21} \mathrm{~F}_{3} \mathrm{NO}_{3} \mathrm{~S}^{+}$ 520.1180; observed 520.1190 .
4-(Dimethylamino)-2-methylene-5-((4-methylnaphthalen-1-yl)methyl)-7-oxo-2,2a-dihydro-7H-cyclobuta[4,5]thiazolo[3,2-a]pyridine- $8 a(1 \mathrm{H})$-carboxylic Acid (6c). The reaction was performed on $20 \mathrm{mg}(0.044 \mathrm{mmol})$ of $5 \mathbf{k}$ in THF $(4 \mathrm{~mL})$ and showed completion in $5 \mathrm{~h}$. Colorless powder, $8 \mathrm{mg}, 41 \%$. IR (KBr): $\nu 3437$, 2925, 2854, 2828, 2786, 1726, 1622, 1531, 1480,1412, 1293, 1216 $\mathrm{cm}^{-1} .{ }^{1} \mathrm{H}$ NMR $\left[400 \mathrm{MHz},\left(\mathrm{CD}_{3}\right)_{2} \mathrm{SO}\right] \delta 8.10-8.01(\mathrm{~m}, 1 \mathrm{H}), 7.93-$ $7.85(\mathrm{~m}, 1 \mathrm{H}), 7.61-7.51(\mathrm{~m}, 2 \mathrm{H}), 7.39-7.24(\mathrm{~m}, 2 \mathrm{H}), 5.28(\mathrm{t}, J=$ $2.7 \mathrm{~Hz}, 1 \mathrm{H}), 5.19(\mathrm{~d}, J=1.1 \mathrm{~Hz}, 1 \mathrm{H}), 5.13(\mathrm{dd}, J=11.6,2.7 \mathrm{~Hz}, 2 \mathrm{H})$, $4.28(\mathrm{~s}, 2 \mathrm{H}), 3.72-3.60(\mathrm{~m}, 1 \mathrm{H}), 2.86(\mathrm{~d}, J=2.6 \mathrm{~Hz}, 1 \mathrm{H}), 2.75(\mathrm{~s}$, $6 \mathrm{H}), 2.66(\mathrm{~s}, 3 \mathrm{H}) .{ }^{13} \mathrm{C}\left\{{ }^{1} \mathrm{H}\right\}$ NMR $\left[151 \mathrm{MHz},\left(\mathrm{CD}_{3}\right)_{2} \mathrm{SO}\right] \delta 168.9$, $159.3,157.4,149.4,133.6,132.9,132.0,128.0,126.7,126.5,126.1$, 125.5, 125.3, 125.0, 113.8, 112.7, 51.8, 43.2, 42.3, 34.2, 19.5. HRMS (ESI-TOF) $m / z:[\mathrm{M}+\mathrm{H}]^{+}$calcd for $\mathrm{C}_{25} \mathrm{H}_{25} \mathrm{~N}_{2} \mathrm{O}_{3} \mathrm{~S}+433.1580$; observed 433.1588 .

Preparation of Imidazolium Carboxylate Salts $8 \boldsymbol{a}-\boldsymbol{b}$. Caboxylicacid $6 \mathbf{a}(8.00 \mathrm{mg}, 0.019 \mathrm{mmol}, 1.0$ equiv) or $\mathbf{6 b}(10.0 \mathrm{mg}, 0.019$ mmol, 1.0 equiv) was dissolved in methanol $(5 \mathrm{~mL})$. Imidazole solution $(20 \mathrm{mg} / \mathrm{mL}$ in methanol, $66 \mu \mathrm{L}, 0.019 \mathrm{mmol}, 1.0$ equiv) was added. After $24 \mathrm{~h}$ of stirring at room temperature, the reaction mixture was concentrated on a rotary evaporator. The residue was dissolved in acetonitrile/water 1:3 $(10 \mathrm{~mL})$ and lyophilized.

General Procedure for Synthesis of $9 \boldsymbol{a}-\boldsymbol{b}$ and 7. The methyl ester $(\approx 0.1 \mathrm{mmol}, 1.0$ equiv) and $\mathrm{LiOH}(0.6 \mathrm{mmol}, 6.0$ equiv) were dissolved in THF/ $\mathrm{H}_{2} \mathrm{O}$ 3:1 $(5 \mathrm{~mL})$ and stirred at room temperature until complete or almost complete hydrolysis of the methyl ester was indicated by TLC analysis. Then, $1 \mathrm{M} \mathrm{HCl}(0.7 \mathrm{mmol}, 7.0$ equiv) was added, and the resulting mixture was stirred for $1 \mathrm{~min}$ or until no further color change was seen. The mixture was evaporated partially (THF) and partitioned between brine $(5 \mathrm{~mL})$ and $\mathrm{DCM} / \mathrm{MeOH}$ 9:1 $(2 \times 10 \mathrm{~mL})$. The organic phase was dried, filtered, and evaporated. The residue was dissolved in DMSO (1-2 mL), filtered through a $0.45 \mu \mathrm{m}$ syringe filter, and purified with preparative reverse phase chromatoghraphy. The fractions containing the pure desired product were combined and concentrated partially and then redissolved by addition of a small amount of $\mathrm{MeCN}$. The solution was diluted by quick addition of water, frozen in liquid nitrogen, and freeze-dried. Note: The hydrolysis of the ring opened products 2 was slower and lower yielding, and the conversion was much less clean compared to general thiazolino fused 2-pyridones and the ring closed compounds 5 .

2-(5-Cyclopropyl-6-(methylthio)-4-(naphthalen-1-ylmethyl)-2oxopyridin-1(2H)-yl)acrylic Acid (9a). The compound was prepared from $2 \mathrm{c}(52 \mathrm{mg}, 0.128 \mathrm{mmol})$ following the general procedure. The reaction was finished after $2.5 \mathrm{~h}$, and the product was subsequently isolated as a white powder $(11 \mathrm{mg}, 0.028 \mathrm{mmol}, 22 \%)$. IR (KBr): $\nu$ $3431,3063,3005,2925,1718,1647,1598,1556,1481,1409,1277$, 1194, 1140, $781 \mathrm{~cm}^{-1} .{ }^{1} \mathrm{H}$ NMR $\left[600 \mathrm{MHz},\left(\mathrm{CD}_{3}\right)_{2} \mathrm{SO}\right] \delta 13.14$ (bs, $1 \mathrm{H}), 8.03-7.94(\mathrm{~m}, 1 \mathrm{H}), 7.89(\mathrm{t}, J=8.4 \mathrm{~Hz}, 2 \mathrm{H}), 7.60-7.47(\mathrm{~m}$, $3 \mathrm{H}), 7.41(\mathrm{~d}, J=6.9 \mathrm{~Hz}, 1 \mathrm{H}), 6.46(\mathrm{~s}, 1 \mathrm{H}), 5.79(\mathrm{~s}, 1 \mathrm{H}), 5.47(\mathrm{~s}$, $1 \mathrm{H}), 4.61-4.44(\mathrm{~m}, 2 \mathrm{H}), 2.39(\mathrm{~s}, 3 \mathrm{H}), 1.83$ (ddd, $J=13.9,8.1,5.8$ $\mathrm{Hz}, 1 \mathrm{H}), 1.23-1.13(\mathrm{~m}, 1 \mathrm{H}), 1.13-1.06(\mathrm{~m}, 1 \mathrm{H}), 0.97(\mathrm{dd}, J=9.6$, $4.5 \mathrm{~Hz}, 1 \mathrm{H}), 0.80-0.70(\mathrm{~m}, 1 \mathrm{H}) .{ }^{13} \mathrm{C}\left\{{ }^{1} \mathrm{H}\right\}$ NMR $[151 \mathrm{MHz}$, $\left.\left(\mathrm{CD}_{3}\right)_{2} \mathrm{SO}\right] \delta 163.8,160.7,156.0,144.8,134.3,133.5,131.6,128.7$, $127.8,127.4,126.4,125.9,125.7,124.0,123.1,118.1,35.6,19.9,11.3$, 10.8, 9.0. HRMS (ESI-TOF) $m / z:[\mathrm{M}+\mathrm{H}]^{+}$calcd for $\mathrm{C}_{23} \mathrm{H}_{22} \mathrm{NO}_{3} \mathrm{~S}^{+}$ 392.1315; observed 392.1324.

2-(5-Cyclopropyl-6-(methylthio)-2-(4-nitrophenyl)-8-oxo-4-phenyl-1,7-naphthyridin-7(8H)-yl)acrylic Acid (7). The compound was prepared from $4(50 \mathrm{mg}, 0.097 \mathrm{mmol})$ following the general procedure. The reaction was finished after $2.5 \mathrm{~h}$, and the product was subsequently isolated as a yellow powder $(14 \mathrm{mg}, 0.028 \mathrm{mmol}$, $29 \%)$. IR (KBr): $\nu 3433,3081,3005,1731,1649,1584,1520,1455$, 1440,1410, 1345, 1312, 1258, 1165, 1144, 854, $736 \mathrm{~cm}^{-1}$. ${ }^{1} \mathrm{H}$ NMR $\left[600 \mathrm{MHz},\left(\mathrm{CD}_{3}\right)_{2} \mathrm{SO}\right] \delta 13.30(\mathrm{bs}, 1 \mathrm{H}), 8.59(\mathrm{~d}, J=8.9 \mathrm{~Hz}, 2 \mathrm{H})$, $8.46-8.29(\mathrm{~m}, 3 \mathrm{H}), 7.82-7.39(\mathrm{~m}, 5 \mathrm{H}), 6.66(\mathrm{~s}, 1 \mathrm{H}), 6.07(\mathrm{~s}, 1 \mathrm{H})$, $2.45(\mathrm{~s}, 3 \mathrm{H}), 1.18$ (ddd, $J=13.9,7.6,6.0 \mathrm{~Hz}, 1 \mathrm{H}), 0.52-0.01(\mathrm{~m}$, $4 \mathrm{H}) .{ }^{13} \mathrm{C}\left\{{ }^{1} \mathrm{H}\right\}$ NMR $\left[151 \mathrm{MHz},\left(\mathrm{CD}_{3}\right)_{2} \mathrm{SO}\right] \delta 164.0,159.3,151.7$, $148.6,148.0,144.5,143.5,142.4,140.7,137.0,133.3,129.2,128.3$, 128.2, 128.0, 127.2, 126.4, 124.0, 118.8, 19.7, 16.5, 13.0, 12.1. HRMS 
(ESI-TOF) $m / z:[\mathrm{M}+\mathrm{H}]^{+}$calcd for $\mathrm{C}_{27} \mathrm{H}_{22} \mathrm{~N}_{3} \mathrm{O}_{5} \mathrm{~S}^{+}$500.1275; observed 500.1286.

2-(6-(Butylthio)-5-cyclopropyl-4-(naphthalen-1-ylmethyl)-2-oxopyridin-1(2H)-yl)acrylic Acid (9b). The compound was prepared from 2f (44 mg, $0.098 \mathrm{mmol}$ ) following the general procedure. The reaction was finished after $3 \mathrm{~h}$, and the product was subsequently isolated as a white powder (12 $\mathrm{mg}, 0.028 \mathrm{mmol}, 28 \%)$. IR (KBr): $\nu$ 3045, 3004, 2958, 2931, 2871, 1720, 1646, 1598, 1480, 1409, 1274, 1194, 1141, 793, $781 \mathrm{~cm}^{-1} .{ }^{1} \mathrm{H}$ NMR [600 MHz, $\left.\left(\mathrm{CD}_{3}\right)_{2} \mathrm{SO}\right] \delta 13.11$ (bs, 1H), 8.04-7.93 (m, 1H), 7.89 (dd, $J=8.7,4.2 \mathrm{~Hz}, 2 \mathrm{H}), 7.61-$ $7.45(\mathrm{~m}, 3 \mathrm{H}), 7.39(\mathrm{~d}, J=7.0 \mathrm{~Hz}, 1 \mathrm{H}), 6.45(\mathrm{~s}, 1 \mathrm{H}), 5.75(\mathrm{~s}, 1 \mathrm{H})$, $5.58(\mathrm{~s}, 1 \mathrm{H}), 4.53(\mathrm{~s}, 2 \mathrm{H}), 2.87(\mathrm{q}, J=7.3 \mathrm{~Hz}, 2 \mathrm{H}), 1.72$ (ddd, $J=$ 13.8, 8.1, 5.6 Hz, $1 \mathrm{H}), 1.46(\mathrm{p}, J=7.3 \mathrm{~Hz}, 2 \mathrm{H}), 1.33$ (ddt, $J=13.9$, 11.3, $6.7 \mathrm{~Hz}, 2 \mathrm{H}), 1.14(\mathrm{tt}, J=8.6,4.3 \mathrm{~Hz}, 1 \mathrm{H}), 1.05(\mathrm{tt}, J=8.4,4.7$ $\mathrm{Hz}, 1 \mathrm{H}), 0.92(\mathrm{dq}, J=10.5,5.4 \mathrm{~Hz}, 1 \mathrm{H}), 0.86(\mathrm{t}, J=7.3 \mathrm{~Hz}, 3 \mathrm{H})$, $0.74(\mathrm{dq}, J=10.1,5.1 \mathrm{~Hz}, 1 \mathrm{H}) .{ }^{13} \mathrm{C}\left\{{ }^{1} \mathrm{H}\right\}$ NMR $[151 \mathrm{MHz}$, $\left.\left(\mathrm{CD}_{3}\right)_{2} \mathrm{SO}\right] \delta 163.7,160.8,155.7,143.5,134.6,133.5,131.5,128.7$, $127.5,127.4,126.4,125.9,125.7,123.9,123.5,118.2,99.5,79.2,35.8$, 35.6, 30.7, 21.2, 13.5, 11.4, 11.0, 9.2. HRMS (ESI-TOF) $\mathrm{m} / \mathrm{z}:[\mathrm{M}+$ $\mathrm{H}]^{+}$calcd for $\mathrm{C}_{26} \mathrm{H}_{28} \mathrm{NO}_{3} \mathrm{~S}^{+}$434.1784; observed 434.1793.

General Procedure for Synthesis of 10a-e. Thiazolino fused 2pyridone $3 \mathrm{a}-\mathrm{e}(0.25 \mathrm{mmol}, 1.0$ equiv) and cesium carbonate $(0.5$ mmol, 2.0 equiv) were weighed together in a $2-5 \mathrm{~mL}$ microwave reaction tube and flushed with nitrogen. Dry THF $(1.5 \mathrm{~mL})$ and propargyl bromide ( $1.05 \mathrm{mmol}, 4.2$ equiv) were added. After $24 \mathrm{~h}$, additional cesium carbonate and propargyl bromide was added, as specified below, and the reaction mixture was left stirring for $1-3 \mathrm{~h}$ more until reaction completion. THF was removed on a rotary evaporator, and the remaining mixture was partitioned between DCM $(50 \mathrm{~mL})$ and brine $(30 \mathrm{~mL})$. The organic phase was filtered, concentrated, and purified with automated flash column chromatography using a $50 \mathrm{~g}$ Sfär cartridge. Because of partial transesterification from methyl ester to propargyl ester and difficulty in their separation by column chromatography, the mixture of both esters was proceeded for ester hydrolysis using $\mathrm{LiOH}$.

General Procedure for Ester Hydrolysis. The obtained mixture of esters was dissolved in THF $(3 \mathrm{~mL})$, and $\mathrm{LiOH}(0.10 \mathrm{M}, 10.0$ equiv) was added. Upon completion, $\mathrm{HCl}(1.00 \mathrm{M}, 11.0$ equiv) was added. The mixture was stirred for $1 \mathrm{~min}$ and concentrated on a rotary evaporator. The residue was dissolved in EtOAc $(50 \mathrm{~mL})$ and washed with brine $(30 \mathrm{~mL})$. The organic phase was concentrated on a rotary evaporator, dissolved in DMSO, filtered, and purified with preparative HPLC. 10a and 10c were instead purified with normal phase chromatography using $5-30 \% \mathrm{MeOH}$ in DCM. The yields are reported as overall yields for the two steps.

5-Cyclopropyl-7-methylene-2-(4-nitrophenyl)-10-oxo-4-phenyl7,8-dihydro-10H-cyclobuta[4,5] thiazolo[2,3-g][1,7]naphthyridine$8 a(6 a H)$-carboxylic Acid (10a). After $24 \mathrm{~h}, \mathrm{Cs}_{2} \mathrm{CO}_{3}(81 \mathrm{mg}, 0.25$ mmol, 1.0 equiv) and propargyl bromide $(56 \mu \mathrm{L}, 0.50 \mathrm{mmol}, 2.0$ equiv) were added and the mixture was stirred for $1 \mathrm{~h}$ more; the reaction completed in $25 \mathrm{~h}$ in total. Reaction residue was purified by automated flash column chromatography (50 g Sfär cartridge, 10$80 \%$ EtOAc in heptane) to give the mixture of esters as a yellow syrup which was subjected to ester hydrolysis by $\mathrm{LiOH}$ in $4 \mathrm{~h}$, as described above. Yellow powder, $20 \mathrm{mg}$, 26\%. IR (KBr): $\nu$ 3725, 2997, 1918, $1584,1549,1520,1458,1376,1344,1281,1197,1109,1034 \mathrm{~cm}^{-1}$. ${ }^{1} \mathrm{H}$ NMR $\left[600 \mathrm{MHz},\left(\mathrm{CD}_{3}\right)_{2} \mathrm{SO}\right] \delta 8.58-8.52(\mathrm{~m}, 2 \mathrm{H}), 8.39-8.33$ $(\mathrm{m}, 2 \mathrm{H}), 8.26(\mathrm{~s}, 1 \mathrm{H}), 7.62-7.56(\mathrm{~m}, 2 \mathrm{H}), 7.51-7.42(\mathrm{~m}, 3 \mathrm{H}), 5.30$ $(\mathrm{s}, 1 \mathrm{H}), 5.18(\mathrm{~s}, 2 \mathrm{H}), 3.84(\mathrm{dt}, J=17.1,2.6 \mathrm{~Hz}, 1 \mathrm{H}), 3.12(\mathrm{dq}, J=$ $17.3,2.6 \mathrm{~Hz}, 1 \mathrm{H}), 1.16-1.12(\mathrm{~m}, 1 \mathrm{H}), 0.16-0.14(\mathrm{~m}, 4 \mathrm{H}) .{ }^{13} \mathrm{C}\left\{{ }^{1} \mathrm{H}\right\}$ NMR $\left[151 \mathrm{MHz},\left(\mathrm{CD}_{3}\right)_{2} \mathrm{SO}\right] \delta 168.6,157.6,150.0,147.8,147.3$, $143.5,140.8,140.5,133.3,129.3,127.9,127.9,127.7,126.6,123.9$, $112.1,107.0,51.0,15.7,10.9,10.8$. HRMS (ESI-TOF) $\mathrm{m} / z:[\mathrm{M}+$ $\mathrm{H}]^{+}$calcd for $\mathrm{C}_{29} \mathrm{H}_{22} \mathrm{~N}_{3} \mathrm{O}_{5} \mathrm{~S}^{+}$524.1275; observed 524.1274.

5-Cyclopropyl-7-methylene-10-oxo-4-phenyl-2-(p-tolyl)-7,8-dihydro-10H-cyclobuta [4,5] thiazolo[2,3-g][1,7]naphthyridine$8 a(6 a H)$-carboxylic Acid (10b). After $24 \mathrm{~h}, \mathrm{Cs}_{2} \mathrm{CO}_{3}(162 \mathrm{mg}, 0.5$ mmol, 2.0 equiv) and propargyl bromide $(113 \mu \mathrm{L}, 1.0 \mathrm{mmol}, 4.0$ equiv) were added and the mixture was stirred for $3 \mathrm{~h}$ more; the reaction completed in $27 \mathrm{~h}$ in total. The crude product was purified by automated flash column chromatography (50 g Sfär cartridge, 10$80 \%$ EtOAc in heptane) to give a mixture of esters as a yellow syrup which was subjected to ester hydrolysis by $\mathrm{LiOH}$ in $7 \mathrm{~h}$, as described above. Light yellow powder, $20 \mathrm{mg}, 16 \%$. IR (KBr): $\nu$ 3358, 1728, $1644,1588,1554,1505,1488,1459,1441,1376,1278,1225,1185$, $1139,1032 \mathrm{~cm}^{-1} .{ }^{1} \mathrm{H}$ NMR $\left[600 \mathrm{MHz},\left(\mathrm{CD}_{3}\right)_{2} \mathrm{SO}\right] \delta 8.16(\mathrm{~s}, 1 \mathrm{H})$, $8.15(\mathrm{~s}, 1 \mathrm{H}), 8.03(\mathrm{~s}, 1 \mathrm{H}), 7.55(\mathrm{~s}, 1 \mathrm{H}), 7.54(\mathrm{~s}, 1 \mathrm{H}), 7.47-7.43(\mathrm{~m}$, $4 \mathrm{H}), 7.33(\mathrm{~s}, 1 \mathrm{H}), 7.32(\mathrm{~s}, 1 \mathrm{H}), 5.30(\mathrm{~s}, 1 \mathrm{H}), 5.22-5.13(\mathrm{~m}, 2 \mathrm{H})$, $3.83(\mathrm{dt}, J=17.0,2.7 \mathrm{~Hz}, 1 \mathrm{H}), 3.10(\mathrm{dt}, J=17.0,2.7 \mathrm{~Hz}, 1 \mathrm{H}), 2.37(\mathrm{~s}$, $4 \mathrm{H}), 1.14-1.10(\mathrm{~m}, 1 \mathrm{H}), 0.16-0.11(\mathrm{~m}, 4 \mathrm{H}) .{ }^{13} \mathrm{C}\left\{{ }^{1} \mathrm{H}\right\}$ NMR $[151$ $\left.\mathrm{MHz},\left(\mathrm{CD}_{3}\right)_{2} \mathrm{SO}\right] \delta 168.8,157.8,152.7,147.0,146.8,145.7,141.1$, $140.3,139.1,134.8,132.3,129.4,127.8,127.7,126.7,125.5,112.2$, $107.2,73.3,51.0,20.8,15.8,10.8,10.7$. HRMS (ESI-TOF) $m / z:[\mathrm{M}+$ $\mathrm{H}]^{+}$calcd for $\mathrm{C}_{30} \mathrm{H}_{25} \mathrm{~N}_{2} \mathrm{O}_{3} \mathrm{~S}^{+}$493.1580; observed 493.1582.

2-(4-Fluorophenyl)-7-methylene-10-oxo-5-phenyl-7,8-dihydro$10 \mathrm{H}$-cyclobuta[4,5]thiazolo[2,3-g][1,7]naphthyridine-8a(6aH)-carboxylic Acid (10c). After $24 \mathrm{~h}, \mathrm{Cs}_{2} \mathrm{CO}_{3}(162 \mathrm{mg}, 0.5 \mathrm{mmol}, 2.0$ equiv) and propargyl bromide ( $113 \mu \mathrm{L}, 1.0 \mathrm{mmol}, 4.0$ equiv) were added and the reaction mixture was stirred for $3 \mathrm{~h}$ more; the reaction completed in $27 \mathrm{~h}$ in total. The crude product was purified by automated flash column chromatography (50 g Sfär cartridge, 10$80 \%$ EtOAc in heptane) to give a mixture of esters as a yellow syrup which was subjected to ester hydrolysis by $\mathrm{LiOH}$ in $8.5 \mathrm{~h}$, as described above. Light yellow powder, $18 \mathrm{mg}, 24 \%$. IR (KBr): $\nu$ 3741, 3508, $1740,1614,1585,1563,1526,1478,1382,1351,1262,1226,1197$, $1186,1171 \mathrm{~cm}^{-1}$. ${ }^{1} \mathrm{H}$ NMR $\left[400 \mathrm{MHz},\left(\mathrm{CD}_{3}\right)_{2} \mathrm{SO}\right] \delta 8.32(\mathrm{dd}, J=$ 8.7, $5.6 \mathrm{~Hz}, 2 \mathrm{H}), 8.08(\mathrm{~s}, 1 \mathrm{H}), 7.57-7.55(\mathrm{~m}, 2 \mathrm{H}), 7.48-7.41(\mathrm{~m}$, $3 \mathrm{H}), 7.35(\mathrm{t}, J=8.7 \mathrm{~Hz}, 2 \mathrm{H}), 5.31(\mathrm{~s}, 1 \mathrm{H}), 5.19(\mathrm{q}, J=2.8 \mathrm{~Hz}, 2 \mathrm{H})$, $3.83(\mathrm{dt}, J=17.1,2.8 \mathrm{~Hz}, 1 \mathrm{H}), 3.10(\mathrm{dt}, J=17.2,2.6 \mathrm{~Hz}, 1 \mathrm{H}), 1.18-$ $1.09(\mathrm{~m}, 1 \mathrm{H}), 0.16-0.09(\mathrm{~m}, 4 \mathrm{H}) .{ }^{13} \mathrm{C}\left\{{ }^{1} \mathrm{H}\right\}$ NMR $[100 \mathrm{MHz}$, $\left.\left(\mathrm{CD}_{3}\right)_{2} \mathrm{SO}\right] \delta 168.8,164.3,161.8,157.8,151.7,147.2,147.1,145.6$, 141.0, 140.3, 134.1, 134.0, 132.4, 129.3, 129.1, 129.0, 127.8, 127.7, $125.7,115.7,115.5,112.2,107.1,99.5,73.3,51.0,15.8,10.8,10.7 .{ }^{19} \mathrm{~F}$ NMR $\left[400 \mathrm{MHz},\left(\mathrm{CD}_{3}\right)_{2} \mathrm{SO}\right] \delta-112.37$. HRMS (ESI-TOF) $\mathrm{m} / z$ : $[\mathrm{M}+\mathrm{H}]^{+}$calcd for $\mathrm{C}_{29} \mathrm{H}_{22} \mathrm{FN}_{2} \mathrm{O}_{3} \mathrm{~S}^{+}$497.1330; observed 497.1326.

5-Cyclopropyl-7-methylene-2-(4-nitrophenyl)-10-oxo-7,8-dihydro-10H-cyclobuta[4,5] thiazolo[2,3-g][1,7]naphthyridine-8a(6aH)carboxylic Acid (10d). After $24 \mathrm{~h}, \mathrm{Cs}_{2} \mathrm{CO}_{3}(162 \mathrm{mg}, 0.5 \mathrm{mmol}, 2.0$ equiv) and propargyl bromide ( $113 \mu \mathrm{L}, 1.0 \mathrm{mmol}, 4.0$ equiv) were added and the mixture was stirred for $24 \mathrm{~h}$ more; the reaction completed in $48 \mathrm{~h}$ in total. After purification, the mixture of esters was subjected to ester hydrolysis for $8 \mathrm{~h}$ as described above. Yellow powder, $15 \mathrm{mg}, 13 \%$. IR (KBr): $\nu$ 3749, 3522, 2897, 1737, 1631, $1589,1572,1524,1474,1412,1377,1338,1280,1223,1186,1157$, $1107,1061,1035 \mathrm{~cm}^{-1} .{ }^{1} \mathrm{H}$ NMR $\left[600 \mathrm{MHz},\left(\mathrm{CD}_{3}\right)_{2} \mathrm{SO}\right] \delta 8.54-$ $8.46(\mathrm{~m}, 5 \mathrm{H}), 8.41-8.37(\mathrm{~m}, 3 \mathrm{H}), 5.31(\mathrm{q}, J=2.8 \mathrm{~Hz}, 1 \mathrm{H}), 5.24(\mathrm{q}, J$ $=2.5 \mathrm{~Hz}, 1 \mathrm{H}), 5.17(\mathrm{q}, J=2.6 \mathrm{~Hz}, 1 \mathrm{H}), 3.82(\mathrm{dt}, J=17.1,2.8 \mathrm{~Hz}$, $1 \mathrm{H}), 3.07(\mathrm{tt}, J=17.0,2.6 \mathrm{~Hz}, 1 \mathrm{H}), 1.87-1.82(\mathrm{~m}, 1 \mathrm{H}), 1.14-1.07$ $(\mathrm{m}, 2 \mathrm{H}), 0.69-0.57(\mathrm{~m}, 2 \mathrm{H}) .{ }^{13} \mathrm{C}\left\{{ }^{1} \mathrm{H}\right\}$ NMR $\left[151 \mathrm{MHz},\left(\mathrm{CD}_{3}\right)_{2} \mathrm{SO}\right]$ $\delta$ 168.7, 157.9, 151.0, 147.8, 143.7, 139.1, 134.8, 133.0, 127.7, 127.7, $124.3,124.1,124.0,112.3,106.7,73.0,51.2,9.7,7.5$, 7.3. HRMS (ESITOF) $\mathrm{m} / z$ : $[\mathrm{M}+\mathrm{H}]^{+}$calcd for $\mathrm{C}_{23} \mathrm{H}_{18} \mathrm{~N}_{3} \mathrm{O}_{5} \mathrm{~S}^{+}$448.0962; observed 448.0968 .

5-Cyclopropyl-7-methylene-10-oxo-2-(p-tolyl)-7,8-dihydro-10Hcyclobuta[4,5]thiazolo[2,3-g][1,7]naphthyridine-8a(6aH)-carboxylic Acid (10e). After $24 \mathrm{~h}, \mathrm{Cs}_{2} \mathrm{CO}_{3}(162 \mathrm{mg}, 0.5 \mathrm{mmol}, 2.0$ equiv) and propargyl bromide ( $113 \mu \mathrm{L}, 1.0 \mathrm{mmol}, 4.0$ equiv) were added and the mixture was stirred for $1 \mathrm{~h}$ more; the reaction completed in $25 \mathrm{~h}$ in total. The crude mixture was purified by automated flash column chromatography (50 g Sfär cartridge, 10-80\% EtOAc in heptane) to give a mixture of esters as a yellow syrup, which was subjected to ester hydrolysis by $\mathrm{LiOH}$ in $6.5 \mathrm{~h}$, as described above. Light yellow powder, $16 \mathrm{mg}, 15 \%$. IR (KBr): $\nu$ 3876, 3520, 2998, 1784, 1726, 1587, 1533, $1514,1490,1460,1441,1410,1377,1277,1227,1158,1075,1034$ $\mathrm{cm}^{-1} .{ }^{1} \mathrm{H}$ NMR $\left[600 \mathrm{MHz},\left(\mathrm{CD}_{3}\right)_{2} \mathrm{SO}\right] \delta 8.16(\mathrm{~s}, 1 \mathrm{H}), 8.15(\mathrm{~s}, 1 \mathrm{H})$, $8.03(\mathrm{~s}, 1 \mathrm{H}), 7.55(\mathrm{~s}, 1 \mathrm{H}), 7.54(\mathrm{~s}, 1 \mathrm{H}), 7.47-7.43(\mathrm{~m}, 4 \mathrm{H}), 7.33(\mathrm{~s}$, $1 \mathrm{H}), 7.32(\mathrm{~s}, 1 \mathrm{H}), 5.30(\mathrm{~s}, 1 \mathrm{H}), 5.22-5.13(\mathrm{~m}, 2 \mathrm{H}), 3.83(\mathrm{dt}, J=$ 17.0, $2.7 \mathrm{~Hz}, 1 \mathrm{H}), 3.10(\mathrm{dt}, J=17.0,2.7 \mathrm{~Hz}, 1 \mathrm{H}), 2.37$ (s, 4H), 1.14$1.10(\mathrm{~m}, 1 \mathrm{H}), 0.16-0.11(\mathrm{~m}, 4 \mathrm{H}) .{ }^{13} \mathrm{C}\left\{{ }^{1} \mathrm{H}\right\}$ NMR [151 MHz, $\left.\left(\mathrm{CD}_{3}\right)_{2} \mathrm{SO}\right] \delta 168.8,157.8,152.7,147.0,146.8,145.7,141.1,140.3$, 
$139.1,134.8,132.3,129.4,127.8,127.7,126.7,125.5,112.2,107.2$, 73.3, 51.0, 20.8, 15.8, 10.8, 10.7. HRMS (ESI-TOF) $m / z:[\mathrm{M}+\mathrm{H}]^{+}$ calcd for $\mathrm{C}_{24} \mathrm{H}_{21} \mathrm{~N}_{2} \mathrm{O}_{3} \mathrm{~S}^{+}$417.1267; observed 417.1266.

\section{ASSOCIATED CONTENT}

\section{(3) Supporting Information}

The Supporting Information is available free of charge at https://pubs.acs.org/doi/10.1021/acs.joc.1c01875.

Experimental procedures and spectra for characterization of compounds synthesized (PDF)

\section{Accession Codes}

CCDC 2087355 contains the supplementary crystallographic data for this paper. These data can be obtained free of charge via www.ccdc.cam.ac.uk/data_request/cif, or by emailing data_request@ccdc.cam.ac.uk, or by contacting The Cambridge Crystallographic Data Centre, 12 Union Road, Cambridge CB2 1EZ, UK; fax: +44 1223336033.

\section{AUTHOR INFORMATION}

\section{Corresponding Author}

Fredrik Almqvist - Department of Chemistry, Umeå

University, 90187 Umeå, Sweden; ๑ orcid.org/0000-0003-

4646-0216; Phone: +46 (0)90 7866925;

Email: fredrik.almqvist@umu.se

\section{Authors}

Mohit Tyagi - Department of Chemistry, Umeå University, 90187 Umeå, Sweden

Dan E. Adolfsson - Department of Chemistry, Umeå University, 90187 Umeå, Sweden

Pardeep Singh - Department of Chemistry, Umeå University, 90187 Umeå, Sweden

Jörgen Ådén - Department of Chemistry, Umeå University, 90187 Umeå, Sweden

Sanduni Wasana Jayaweera - Department of Medical Biochemistry and Biophysics, Umeå University, 90187 Umeå, Sweden

Anna Gharibyan - Department of Medical Biochemistry and Biophysics, Umeå University, 90187 Umeå, Sweden

Jaideep B. Bharate - Department of Chemistry, Umeå University, 90187 Umeå, Sweden; @ orcid.org/0000-00033269-6228

Anita Kiss - Department of Chemistry, Umeå University, 90187 Umeå, Sweden

Souvik Sarkar - Department of Chemistry, Umeå University, 90187 Umeå, Sweden

Anders Olofsson - Department of Medical Biochemistry and Biophysics, Umeå University, 90187 Umeå, Sweden

Complete contact information is available at: https://pubs.acs.org/10.1021/acs.joc.1c01875

\section{Author Contributions}

${ }^{\S}$ M.T., D.E.A., and P.S. contributed equally to this work.

\section{Notes}

The authors declare the following competing financial interest(s): F.A. has ownership interests in Quretech Bio AB. The other authors have no competing financial interest.

\section{ACKNOWLEDGMENTS}

F.A. is grateful to the Swedish Research Council (2017-02339, 2017-00695, and 2018-04589), the Knut and Alice Wallenberg Foundation (KAW 2013.0031), the Göran Gustafsson
Foundation, the Kempe Foundation (SMK-1755), the Swedish Foundation for Strategic Research (SB12-0070), the National Institutes of Health (R01AI134847-01A1), the Erling Perssons Stiftelse, and the Michael J. Fox Foundation for financial support. This project has been supported under the framework of the JPIAMR - Joint Programming Initiative on Antimicrobial Resistance. We thank Prof. Ola F. Wendt at Lund University for obtaining the X-ray crystal structure.

\section{REFERENCES}

(1) Morrison, C. N.; Prosser, K. E.; Stokes, R. W.; Cordes, A.; Metzler-Nolte, N.; Cohen, S. M. Expanding medicinal chemistry into 3D space: Metallofragments as 3D scaffolds for fragment-based drug discovery. Chem. Sci. 2020, 11, 1216-1225.

(2) (a) Liu, Q.; Li, N.; Yuan, Y.; Lu, H.; Wu, X.; Zhou, C.; He, M.; Su, H.; Zhang, M.; Wang, J.; Wang, B.; Wang, Y.; Ma, D.; Ye, Y.; Weiss, H. C.; Gesing, E. R.; Liao, J.; Wang, M. W. Cyclobutane derivatives as novel nonpeptidic small molecule agonists of glucagonlike peptide-1 receptor. J. Med. Chem. 2012, 55, 250-267. (b) Marson, C. M. New and unusual scaffolds in medicinal chemistry. Chem. Soc. Rev. 2011, 40, 5514-5533. (c) Oderinde, M. S.; Ramirez, A.; Dhar, T. G. M.; Cornelius, L. A. M.; Jorge, C.; Aulakh, D.; Sandhu, B.; Pawluczyk, J.; Sarjeant, A. A.; Meanwell, N. A.; Mathur, A.; Kempson, J. Photocatalytic Dearomative Intermolecular $[2+2]$ Cycloaddition of Heterocycles for Building Molecular Complexity. J. Org. Chem. 2021, 86, 1730-1747. (d) Poplata, S.; Tröster, A.; Zou, Y. Q.; Bach, T. Recent Advances in the Synthesis of Cyclobutanes by Olefin [2+ 2] Photocycloaddition Reactions. Chem. Rev. 2016, 116, 9748-9815. (e) Narasaka, K.; Hayashi, Y.; Shimadzu, H.; Niihata, S. Asymmetric $[2+2]$ cycloaddition reaction catalysed by a chiral titanium reagent. J. Am. Chem. Soc. 1992, 114, 8869-8885.

(3) Ishihara, K.; Nakano, K. Enantioselective $[2+2]$ cycloaddition of unactivated alkenes with alpha-acyloxyacroleins catalyzed by chiral organoammonium salts. J. Am. Chem. Soc. 2007, 129, 8930-8931.

(4) Chapman, L. M.; Beck, J. C.; Wu, L.; Reisman, S. E. Enantioselective Total Synthesis of (+)-Psiguadial B. J. Am. Chem. Soc. 2016, 138, 9803-9806.

(5) Liu, J.; Wang, S. M.; Qin, H. L. Light-induced $[2+2]$ cycloadditions for the construction of cyclobutane-fused pyridinyl sulfonyl fluorides. Org. Biomol. Chem. 2020, 18, 4019-4023.

(6) Tröster, A.; Alonso, R.; Bauer, A.; Bach, T. Enantioselective Intermolecular $[2+2]$ Photocycloaddition Reactions of $2(1 \mathrm{H})$ Quinolones Induced by Visible Light Irradiation. J. Am. Chem. Soc. 2016, 138, 7808-7811.

(7) (a) Chorell, E.; Pinkner, J. S.; Phan, G.; Edvinsson, S.; Buelens, F.; Remaut, H.; Waksman, G.; Hultgren, S. J.; Almqvist, F. Design and synthesis of C-2 substituted thiazolo and dihydrothiazolo ring-fused 2pyridones: pilicides with increased antivirulence activity. J. Med. Chem. 2010, 53, 5690-5695. (b) Flentie, K.; Harrison, G. A.; Tükenmez, H.; Livny, J.; Good, J. A. D.; Sarkar, S.; Zhu, D. X.; Kinsella, R. L.; Weiss, L. A.; Solomon, S. D.; Schene, M. E.; Hansen, M. R.; Cairns, A. G.; Kulén, M.; Wixe, T.; Lindgren, A. E. G.; Chorell, E.; Bengtsson, C.; Krishnan, K. S.; Hultgren, S. J.; Larsson, C.; Almqvist, F.; Stallings, C. L. Chemical disarming of isoniazid resistance in Mycobacterium tuberculosis. Proc. Natl. Acad. Sci. U. S. A. 2019, 116, 10510-10517. (c) Good, J. A. D.; Kulén, M.; Silver, J.; Krishnan, K. S.; Bahnan, W.; Núñez-Otero, C.; Nilsson, I.; Wede, E.; de Groot, E.; Gylfe, Å.; Bergström, S.; Almqvist, F. Thiazolino 2-Pyridone Amide Isosteres As Inhibitors of Chlamydia trachomatis Infectivity. J. Med. Chem. 2017, 60, 9393-9399. (d) Kulén, M.; Lindgren, M.; Hansen, S.; Cairns, A. G.; Grundström, C.; Begum, A.; van der Lingen, I.; Brännström, K.; Hall, M.; Sauer, U. H.; Johansson, J.; Sauer-Eriksson, A. E.; Almqvist, F. Structure-Based Design of Inhibitors Targeting PrfA, the Master Virulence Regulator of Listeria monocytogenes. J. Med. Chem. 2018, 61, 4165-4175. (e) Pinkner, J. S.; Remaut, H.; Buelens, F.; Miller, E.; Aberg, V.; Pemberton, N.; Hedenström, M.; Larsson, A.; Seed, P.; Waksman, G.; Hultgren, S. J.; Almqvist, F. Rationally designed small 
compounds inhibit pilus biogenesis in uropathogenic bacteria. Proc. Natl. Acad. Sci. U. S. A. 2006, 103, 17897-17902.

(8) (a) Aberg, V.; Norman, F.; Chorell, E.; Westermark, A.; Olofsson, A.; Sauer-Eriksson, A. E.; Almqvist, F. Microwave-assisted decarboxylation of bicyclic 2-pyridone scaffolds and identification of Abeta-peptide aggregation inhibitors. Org. Biomol. Chem. 2005, 3, 2817-2823. (b) Adolfsson, D. E.; Tyagi, M.; Singh, P.; Deuschmann, A.; Ådén, J.; Gharibyan, A. L.; Jayaweera, S. W.; Lindgren, A. E. G.; Olofsson, A.; Almqvist, F. Intramolecular Povarov Reactions for the Synthesis of Chromenopyridine Fused 2-Pyridone Polyheterocycles Binding to $\alpha$-Synuclein and Amyloid- $\beta$ Fibrils. J. Org. Chem. 2020, 85, 14174-14189. (c) Horvath, I.; Sellstedt, M.; Weise, C.; Nordvall, L. M.; Krishna Prasad, G.; Olofsson, A.; Larsson, G.; Almqvist, F.; Wittung-Stafshede, P. Modulation of $\alpha$-synuclein fibrillization by ringfused 2-pyridones: templation and inhibition involve oligomers with different structure. Arch. Biochem. Biophys. 2013, 532, 84-90. (d) Singh, P.; Adolfsson, D. E.; Ådén, J.; Cairns, A. G.; Bartens, C.; Brännström, K.; Olofsson, A.; Almqvist, F. Pyridine-Fused 2Pyridones via Povarov and A(3) Reactions: Rapid Generation of Highly Functionalized Tricyclic Heterocycles Capable of Amyloid Fibril Binding. J. Org. Chem. 2019, 84, 3887-3903. (e) Singh, P.; Cairns, A. G.; Adolfsson, D. E.; Ådén, J.; Sauer, U. H.; Almqvist, F. Synthesis of Densely Functionalized N-Alkenyl 2-Pyridones via Benzyne-Induced Ring Opening of Thiazolino-Fused 2-Pyridones. Org. Lett. 2019, 21, 6946-6950.

(9) (a) Gulías, M.; Collado, A.; Trillo, B.; López, F.; Oñate, E.; Esteruelas, M. A.; Mascareñas, J. L. Ruthenium-catalyzed $(2+2)$ intramolecular cycloaddition of allenenes. J. Am. Chem. Soc. 2011, 133, 7660-7663. (b) Li, X.; Jandl, C.; Bach, T. Visible-LightMediated Enantioselective Photoreactions of 3-Alkylquinolones with 4-O-Tethered Alkenes and Allenes. Org. Lett. 2020, 22, 3618-3622. (c) Nada, T.; Yoneshige, Y.; Ii, Y.; Matsumoto, T.; Fujioka, H.; Shuto, S.; Arisawa, M. Nonmetathesis Heterocycle Formation by Ruthenium-Catalyzed Intramolecular [2+2] Cycloaddition of Allenamideenes to Azabicyclo [3.1. 1] heptanes. ACS Catal. 2016, 6, 3168-3171. (d) Zhai, S.; Qiu, S.; Chen, L.; Niu, Y.; Yu, Y.; Yang, B.; Zhang, B.; Han, C.; Yang, L.; Zhai, H. Synthesis of cyclobutane-fused oxygencontaining tricyclic framework via thermally promoted intramolecular cycloaddition of cyclohexadienone-tethered allenes. Chem. Commun. (Cambridge, U. K.) 2020, 56, 3405-3408.

(10) (a) Braun, M.-G.; Katcher, M. H.; Doyle, A. G. Carbofluorination via a palladium-catalyzed cascade reaction. Chem. Sci. 2013, 4, 1216-1220. (b) Hu, C.; Chen, Y. Chemoselective and fast decarboxylative allylation by photoredox catalysis under mild conditions. Org. Chem. Front. 2015, 2, 1352-1355. (c) Ma, S.; Hao, X.; Meng, X.; Huang, X. Studies on the regio- and stereoselectivity of halohydroxylation of 1,2-allenyl sulfides or selenides. J. Org. Chem. 2004, 69, 5720-5724. (d) Mitra, D.; Banerjee, D. R.; Das, A. K.; Basak, A. Design, synthesis and reactivity of C2-symmetric azobenzene-based amino acid-bis(propargyl sulfones). Bioorg. Med. Chem. Lett. 2010, 20, 6831-6835.

(11) Chen, G.-f.; Xu, T.-h.; Yan, Y.; Zhou, Y.-r.; Jiang, Y.; Melcher, K.; Xu, H. E. Amyloid beta: structure, biology and structure-based therapeutic development. Acta Pharmacol. Sin. 2017, 38, 1205-1235. 